\title{
Meta-analysis of ridge-furrow cultivation effects on maize production and water
} use efficiency

Yunqi Wang, ${ }^{\mathrm{a}, *}$, Tongji Guo ${ }^{\mathrm{a}}$, Liuran Qi ${ }^{\mathrm{a}}$, Huanyu Zeng ${ }^{\mathrm{a}}$, Yuexin Lianga ${ }^{\mathrm{a}}$, Shikun Weia, Fuli Gao ${ }^{\text {a }}$, Lixin Wang ${ }^{\text {b, }}$, Rui Zhang ${ }^{\text {a, }}$, Zhikuan Jia ${ }^{\text {a, * }}$

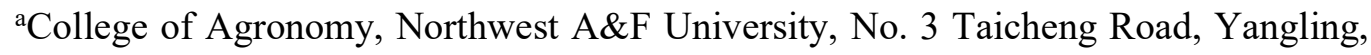
Shaanxi 712100, China

${ }^{b}$ Department of Earth Sciences, Indiana University-Purdue University Indianapolis (IUPUI), Indianapolis, 46202, United States of America

\section{ABSTRACT}

Ridge-furrow cultivation (RF) is a popular dryland agricultural technique in China, but its effects on maize yield, total water consumption during crop growing stage (ET), and water use efficiency (WUE) have not been systematically analyzed. Here we conducted a meta-analysis of the RF effects on maize yield, ET and WUE based on the data collected from peer-reviewed literature. Yield, ET and WUE varied with climate, soil and mulching management. Averaged across all the geographic locations, RF increased the yield and WUE of maize by $47 \%$ and $39 \%$, respectively, but no effects on ET. An increase in the yield and WUE occurred under RF in regions regardless of the mean growing season air temperature (MT) or a mean precipitation during the growing season (MP), although there was a trend that RF is more beneficial under low MP. RF also decreased ET in regions with $\mathrm{MT}>12^{\circ} \mathrm{C}$. RF increased the yield and WUE in regions

\footnotetext{
*Corresponding authors. E-mail address: wyqay163@nwafu.edu.cn (Y. Wang), 1xwang@iupui.edu (L. Wang),zr115@nwafu.edu.cn (R.Zhang),jiazhk@126.com (Z. Jia).
}

This is the author's manuscript of the article published in final edited form as: 
with medium or fine soil texture. RF increased the yield, ET, and WUE in regions with low soil bulk density (BD) $\left(\leq 1.3 \mathrm{~g} \mathrm{~cm}^{-3}\right)$. But in areas where BD is larger than $1.3 \mathrm{~g}$ $\mathrm{cm}^{-3}$, RF only increased the yield and WUE. RF increased the yield and WUE with or without mulching, but decreased ET when no mulching was used. Together, optimizing RF effects on the yield, ET and WUE in maize was largely dependent on environmental conditions and management practices.

Keywords: climate change; precipitation; soil texture; yield; plastic mulching 


\section{Introduction}

Dryland crop production remains the primary source of staple food for the majority of densely-populated regions such as China, sub-Saharan Africa and India (Daryanto et al., 2017). Water supply constraints are recognized as major factors affecting dryland crop production (Wang et al., 2018a), thus dryland crop production is a continuous exercise to allocate the limited rain-water supply to meet the total water consumption during crop growing stage (ET). Therefore, the development of methods to improve agricultural productivity and water use efficiency (WUE) in regions with limited water resources remains crucial (Wu et al., 2015).

By increasing soil water availability and yield, ridge-furrow cultivation (RF) has been widely used in dryland maize cultivation in northwest China since the 1980s (Li et al., 2007; Ren et al., 2008). In the past forty years, many field experiments have been conducted to examine the effects of RF on maize production in China, but the reported effects of RF on maize yield, ET and WUE varied greatly due to different climate, soil factors, and mulching management practices.

There are many studies showing different extents of yield increase in different climatic gradients across China. A study showed RF increased yield, water and precipitation productivity in maize as compared with flat-plot cultivation (FP) under a typical sub-humid drought-prone climate (Yin et al., 2018). An increase in maize yield and water productivity was found under RF in semiarid regions of China (Jia et al., 2018a, b). The RF and plastic-mulching technique provides a potential opportunity of substantially increasing crop yields in semiarid regions of China, but this technology 
brings about a challenge in maintaining soil fertility (Zhou et al., 2012). Wang et al. (2018b) also demonstrated that RF with plastic mulching is an effective droughtresistant farming technology, which has been widely used in the semiarid regions of China, and increased crop yields by more than $30 \%$. One study reported that the RF was combined with irrigation to increase the crop water use efficiency in a semi-humid climate (Wu et al., 2015). The yield and WUE were significantly higher under RF with low fertilizer as compared with high fertilizer and medium fertilizer in wet year. However, in both normal and drought years, the grain yield and WUE were significantly higher under RF with medium fertilizer (Zhang et al., 2018). Another study showed that a significant increase range was observed under the average grain yields in the RF with plastic mulch and high irrigation amount, RF with plastic mulch- and low irrigation amount and RF with high irrigation amount treatments between two years with different precipitation amount (Dong et al., 2018a). These studies indicated that there were various effects of RF on the yield and WUE in different regions resulted from different temperature, precipitation and soil conditions.

Variability in results were also found among different mulching management practices. In southeast Kenya, the grain yield and WUE in RF with plastic mulch treatments were increased as compared with RF without plastic film (Mo et al., 2016). In addition, Liu et al. (2014a) showed that RF with plastic mulch could sustain high grain yields in maize and maintain soil water balance under semiarid environment. The RF with plastic mulch increased grain yield and WUE as compared to no mulch (Li et al., 2013; Liu et al., 2014b). A different increase range in the maize yields was found 
among RF with mulched with plastic film, biodegradable film, maize straw ( $\mathrm{Li}$ et al., 2013). However, where the RF with plastic mulch has been applied in successive years, the annual balance of soil water has been affected and the risk of soil desiccation exacerbated (Wang et al., 2018b), and white pollution. Overall, the effects RF on the yield and WUE varied with mulching management practices.

The impacts of RF on the yield, ET, and WUE in maize mainly depend on several co-varying factors (i.e., agroclimatic regions, soil texture, and mulching management). A meta-analysis showed that soil mulching significantly enhances yields as well as water- and nitrogen-use efficiency of maize and wheat (Qin et al. 2015). Yu et al. (2018) documented benefits and limitations to straw- and plastic-film mulch on maize yield and WUE using a meta-analysis across hydrothermal gradients. By covering the ridges with plastic and channeling rainwater into a very narrow planting zone (furrow), a meta-analysis showed that plastic mulching resulted in a yield increase (Daryanto et al. 2017).

However, the effects of RF on the yield, ET, and WUE of maize have not been quantified across a range of agroecological conditions which incorporate different environmental and management factors. As site-specific field experiments often vary, meta-analysis is useful for summarizing the results from numerous independent experiments on RF (Hedges et al., 1999). Therefore, the main objectives of this study were to conduct a meta-analysis to (1) evaluate the effects of RF on the yield, ET, and WUE of maize, and (2) determine how the effects vary with environmental and mulching management factors. 


\section{Materials and methods}

\subsection{Database}

We searched for articles reporting the impact of RF on maize production and WUE in the arid and semiarid rain-fed areas of China using Web of Science and China National Knowledge Infrastructure. The search only included combinations of the following terms: (i) ridge-furrow, maize, yield, and water use efficiency or (ii) ridge and furrow, corn, yield, and WUE.

We systematically reviewed all results published before February 2019. The articles were included in the database only if they met the following criteria: the studies were monoculture maize or corn (Zea mays L.) sown under field conditions (excluding pot studies and greenhouse experiments) in China. The process was conducted following the flowchart diagram presented in Fig. 1 from Moher et al. (2009), and 22 published articles were used in this study (Table 1 and S1 Information).

The 22 references were then ranked according to the number of citations that they received between 2007 and 2019. Based on the rank, those references were divided into two groups. The literature with the top $50 \%$ citations was the first group while the remaining was the second group. Initial analyses were conducted to compare the responses of yield, ET, and WUE to explanatory variables between the two groups ( $\mathrm{Yu}$ et al., 2015). ET was defined as the total water consumption during crop growing stage $(\mathrm{ET}=$ irrigated water + soil water storage at harvest - soil water storage before sowing + precipitation during the growing season). Meanwhile, WUE is defined as the ratio of yield to ET (WUE = yield / ET). If a reference only has yield, or WUE data, but not ET 
data, the mean precipitation during the growing season (MP) in the rainfed areas was considered as ET. Since our initial analyses showed that the two datasets gave very similar results, subsequent analyses were carried out on the combined sample of 22 references, comprising 24 independent sampling sites, 546 observations of yield, 213 observations of ET, and 438 observations of WUE (Fig. 2 and Tables 1). In all studies, the comparability between control (FP, flat-plot cultivation) and treatments (RF, ridgefurrow cultivation) was established based on either climate, soil characteristics, or mulching managements (Table 1).

Data were either obtained from tables or extracted from figures using GETDATA GRAPH DIGITIZER (v.2.24; Moscow, Russian Federation). When only yield and ET data were provided in articles, the WUE was calculated with the formula WUE $=$ Yield/ET. Standard deviation was used as the measure of variance and it was obtained or calculated from the published measure of variance in each study.

The data were grouped to maximize in-group homogenization. Mean growing season air temperature of maize (MT) was divided into two classes, $\leq 12^{\circ} \mathrm{C},>12^{\circ} \mathrm{C}$ (Wang et al., 2018c). MP of maize was divided into two classes, $\leq 400 \mathrm{~mm},>400 \mathrm{~mm}$. Soil texture in a soil layer with a depth of $0-20 \mathrm{~cm}$ was grouped into three basic classes (coarse, medium, and fine) according to Daryanto et al. (2015). The soil bulk density (BD, dry weight of undisturbed soil per unit volume) in a soil layer with a depth of 0 $20 \mathrm{~cm}$ generally ranges between 1.0 and $1.5 \mathrm{~g} \mathrm{~cm}^{-3}$, and thus $\mathrm{BD}$ was divided into two classes $\left(\leq 1.3 \mathrm{~g} \mathrm{~cm}^{-3},>1.3 \mathrm{~g} \mathrm{~cm}^{-3}\right)$. The field capacity (FC), the amount of water that remains after the excess has drained away from the saturated soil is generally $25 \%$ in a 
soil layer with a depth of $0-20 \mathrm{~cm}$. We then categorized FC into two ranges: $\leq 25 \%$ and $>$ $25 \%$. The mulching management was grouped into three types (RF, ridge-furrow; RFS, ridge-furrow mulched with straw; RFP, ridge-furrow mulched with plastic film).

\subsection{Meta-analysis}

To characterize the response of maize yield, ET, and WUE to RF, a randomeffects meta-analysis was used. We used the natural $\log$ of the response ratio $(\ln R)$ as a measure of effect size:

$$
\ln R=\ln \left(X_{r} / X_{f}\right)=\ln X_{r}-\ln X_{f}
$$

where $X_{r}$ and $X_{f}$ are the measured values of the response variable under RF and FP, respectively (Hedges et al., 1999). Generally, not all of observations are weighted by the inverse of the variance. Individuals with a lower variance should have higher weight.

The sampling variance (e.g., the standard deviation) was not presented in some of the collected studies in our database, but the sample size was reported in all the studied articles. As a result, the $\ln R$ was weighted by sample size, i.e.:

$$
W_{n}=n_{f} n_{r} /\left(n_{f}+n_{r}\right)
$$

where $n_{f}$ and $n_{r}$ are the sample sizes for the FP and RF groups, respectively (Hedges \& Olkin, 1985). The higher weighting is given to well-replicated studies with larger sample sizes under these conditions (Hedges \& Olkin, 1985).

To avoid assigning relatively high weights to those studies for multiple years, the weight of each effect size was divided by the number of years the data from the corresponding study (Wang et al., 2018c). The mean effect sizes were estimated as follows: 


$$
\ln \bar{R}=\sum\left(\ln R_{n} \times W_{n}\right) / \sum W_{n}
$$

where $\ln R_{n}$ is the effect size of the $i$ comparison and $W_{n}$.

The Stata software package (ver. 12.0; Stata Corp., College Station, TX, USA) was used to calculate mean effect sizes and generate bias-corrected $95 \%$ confidence intervals (CIs) for each mean effect size with a metan procedure. If the $95 \%$ bootstrap CIs values did not overlap with zero, a significant RF response was considered. Otherwise, the RF was considered to have no significant impact on yield, ET or WUE under those factors (Hedges et al., 1999). To simplify the interpretation, the effect size (ES, \%) was expressed as the percentage change, which was estimated as follows:

$$
\mathrm{ES}=(\mathrm{R}-1) \times 100 \%
$$

A negative (or positive) percentage change indicated a decrease (or increase) in the response variable under RF relative to FP.

\subsection{Correlation analysis}

The correlation analysis was applied with the PROC CORR procedure (SAS Institute Inc., 2013) to test the relationships between the $\ln R$ of WUE with the $\ln R$ of yield and ET under climate, soil characteristics, or mulching managements. In addition, the correlation analysis was used to detect the relationship between MP, MT, BD, FC with the $\ln R$ of yield, ET and WUE via the PROC CORR procedure (SAS Institute Inc., 2013).

\section{Results}

Averaged across a wide range of environmental and management conditions, RF increased maize yield and WUE by $47 \%$ and $39 \%$, respectively. RF, however, did 
not impact ET (Fig. 3). The $\ln R$ of WUE was significantly and positively related with yield $(P<0.0001)$. There was a significant $(P<0.05)$ negative relationship between the $\ln R$ of WUE and ET (Table 2), indicating that the increase in WUE under RF was related to the increase in yield and decrease in ET.

\subsection{Yield, ET and WUE impacts by climate}

The responses of maize yield, ET, and WUE to RF varied with climate (Fig. 4). Compared with FP, RF significantly increased yield (75\%), ET (9\%) and WUE $(62 \%)$ in regions with an $\mathrm{MT} \leq 12{ }^{\circ} \mathrm{C}$ (Fig. 4A). This increase in WUE (62\%) was similar in regions with an $\mathrm{MT}$ of $>12{ }^{\circ} \mathrm{C}$, although the increase of yield was lower (40\%) and there was a significant decreasing effect of RF on ET (Fig. 4B). In addition, the $\ln R$ of WUE was positively correlated with the $\ln R$ of yield $(P<0.0001)$ regardless of MT, but it was negatively correlated with ET (Table 2). These results indicated that the increase in WUE under RF was due to the increase in yield and the decrease in ET.

RF significantly increased yield (50\%), ET (9\%) and WUE (37\%) in regions with $\mathrm{MP} \leq 400 \mathrm{~mm}$ (Fig. 5A), but the increase was lower in regions with $\mathrm{MP}>400 \mathrm{~mm}$, yield only increased by $16 \%$, ET by $2 \%$ and WUE by $30 \%$ (Fig. $5 \mathrm{~B}$ ). Meanwhile, the $\ln R$ of WUE was significantly positively related with the $\ln R$ of yield, and there was a significant negative correlation between the $\ln R$ of WUE and the $\ln R$ of ET regardless of MP (Table 2), indicating that the increase in WUE was related to the increase in yield and the decrease in ET.

\subsection{Yield, ET and WUE impacts by soil factors}


The effects of RF on maize yield, ET, and WUE varied among soil textures (Fig. 6). RF increased yield by $40 \%$, but not WUE as compared with FP in regions with coarse soil (Fig. 6A). In regions with medium soil texture, RF significantly increased yield (29\%), ET (29\%), and WUE (17\%) as compared with FP (Fig. 6B). Yield and WUE increased by $110 \%$ and $76 \%$ respectively, but no effects of RF on ET compared with FP in regions with fine soil texture (Fig. 6C). Meanwhile, the $\ln R$ of WUE was significantly positively related with the $\ln R$ of yield regardless of soil texture (Table 2).

The effects of RF on yield, ET, and WUE varied with soil bulk density (Fig. 7A and B). RF significantly increased yield, ET, WUE by $69 \%, 5 \%, 57 \%$, respectively, compared with FP in regions with a soil bulk density of $\leq 1.3 \mathrm{~g} \mathrm{~cm}^{-3}$ (Fig. 7A). A lesser extent of increase ( $47 \%$ for yield and $44 \%$ for WUE) was observed in sites with higher bulk density $\left(>1.3 \mathrm{~g} \mathrm{~cm}^{-3}\right)$ where RF also significantly decreased maize ET (9\%) as compared with FP (Fig. 7B). Meanwhile, the $\ln R$ of WUE was significantly positively related with the $\ln R$ of yield regardless of bulk density classification (Table 2).

The responses of yield, ET, and WUE to RF varied by field capacity (Fig. 7C and D). RF significantly increased yield (68\%), ET (50\%) and WUE (46\%), compared with FP in regions with a field capacity of $\leq 25 \%$ (Fig. 7C). We also found a significant increase of $34 \%, 44 \%$ and $30 \%$ in yield, ET and WUE under RF, compared with FP in regions with a field capacity of $>25 \%$ (Fig. 7D). In addition, there was a positive correlation between the $\ln R$ of WUE and the $\ln R$ of yield $(P<0.0001)$ regardless of field capacity, but a negative correlation between the $\ln R$ of WUE and ET only in regions with a field capacity of $\leq 25 \%(P<0.01)$ (Table 2$)$. These results indicated that the 
increase in WUE under RF in regions with a field capacity of $\leq 25 \%$ was due to the increase in yield and the decrease in ET, but the increase in WUE under RF in regions with a field capacity of $>25 \%$ was due to the increase in yield.

\subsection{Yield, ET and WUE impacts by mulching managements}

The effects of RF on maize yield, ET, and WUE varied among mulching managements (Fig. 8). RF significantly increased yield (44\%) and WUE (39\%), but decreased ET (6\%) when no mulching was applied (Fig. 8A). Straw mulching produced similar trends (i.e., $51 \%$ increase in yield and 24\% increase in WUE), but no effects on

ET (Fig. 8B). RF significantly increased yield (48\%) and WUE (40\%), but it did not affect ET when combined with plastic film mulching (Fig. 8C). Meanwhile, the $\ln R$ of WUE was significantly positively related with the $\ln R$ of yield with or without mulching (Table 2).

\section{Discussion}

\subsection{Climate impact}

Our meta-analysis showed the effects of RF on yield, ET and WUE varied with climate (i.e., temperature and precipitation). RF significantly increased soil topsoil temperature during early spring, resulting in the promotion of plant growth (Wang et al., 2015b). Early spring is usually characterized by freezing soil temperature and therefore increasing soil temperature contributes to greater yield under RF. Our results, however, showed that the impacts of RF were positive to yield and WUE regardless of 
MT (Fig. 4 and Fig. S1). The above discussions indicated that the RF yield-increasing effects was due to the increase in soil temperature.

The RF technique has been widely used in rainfed arid and semiarid areas because it: (i) significantly increases soil water storage when precipitation is limited and (ii) reduces surface runoff when rainfall is intense (Li et al., 2013). Although RF in combination with irrigation and limited planting densities can enhance maize water productivity and economic returns under the RF in semi-arid regions of China (Jia et al., 2018a, b), such strategy is only applicable when MP is much lower than normal. Our study indicated the increasing effects of RF yield, ET and WUE decreased as rainfall increased (Fig. 5). El-Sadek \& Salem. (2015) and Muhammad et al. (2017) found that field crops grown under RF usually enhance crop yield between 50 and $100 \%$ in drought years, but only between 10 and $40 \%$ during normal years compared with those grown under FP.

\subsection{Soil impacts}

Soil texture, soil bulk density, and field capacity are the key factors which modulate the effect of RF on soil moisture content and subsequent ET, WUE and crop yield. In most cases, soil texture can provide a good estimate for soil-water potential, water holding capacity, and water availability for plant growth (Daryanto et al., 2016).

Our results showed that the effects of RF on yield, ET, and WUE varied among soil textures (Fig. 6). RF significantly increased maize yield by $40 \%$, but not WUE compared with FP in regions with coarse soil (Fig. 6A). In regions with fine soil, we 
found $110 \%$ and $76 \%$ in yield and WUE, respectively, under RF, but no effects of RF on ET as compared with FP (Fig. 6C).

In addition, soil bulk density and field capacity also affected yield, ET, and WUE under RF. A study showed the soil bulk density affected the morphology and anchorage mechanics of the root systems of sunflower and maize (Goodman et al., 1999). The effects of increased rainwater collection and infiltration were the main advantages of RF (Zhang et al., 2011). Therefore, areas with low soil bulk density or field capacity benefited the most with the application of RF (Fig. 7). These results are also supported by negative correlations between $\ln R$ of yield, ET, or WUE and soil bulk density or field capacity (Table 3 and Fig. S2), suggesting that RF could generate a higher yield increase in yield and WUE in region with low soil bulk density or field capacity.

\subsection{Impacts by mulching managements}

In modern world, different kind of mulching materials are used in crop production, and the yield-increasing-effects vary among different mulching materials (Muhammad et al., 2017). Our study also showed the effects of RF on yield, ET, and WUE varied among RF without mulching, RF mulched with straw and RF mulched with plastic film (Fig. 8). RF mulched with plastic film is an effective method to increase crop WUE and yield in semiarid regions (Zhou et al., 2009). In addition, Daryanto et al. (2017) found that plastic mulching resulted in an increase in yield and a decrease in water consumption as comparable with irrigated crops, due primarily to a 
much greater WUE and better retention of soil water. While plastic mulching can be beneficial in terms of crop yield, plastic also generates serious pollution hazards (Liu et al., 2014c). Biodegradable mulch film and multi-functional mulch recovery machinery are therefore recommended for future uses ( $\mathrm{Ng}$ et al., 2018).

\section{Conclusions}

Our results showed that RF had no effects on ET, but significantly increased WUE by $39 \%$, which contributed to a $47 \%$ increase in maize yield. RF, a practice that is indigenous to China and India and now spreading around the world, is an important and innovative water-saving tool for increasing crop yields and securing food supply in arid and semiarid regions. However, such increase occurs at the expense of a large amount of soil water and fertilizer. Because the responses of maize yield, ET, and WUE to RF varied with climate (precipitation and temperature), soil (soil texture, bulk density, field capacity), and mulching managements. The environmental and management conditions should be considered when promoting the implementation of RF. This synthesis quantified the effects of RF on yield, ET, and WUE based on the available scientific data, providing a basis for promoting the development and improvement of RF maize management under various conditions.

\section{Acknowledgments}

We thank the anonymous reviewers and editors for their constructive comments on this manuscript. We gratefully acknowledge financial support for this research from the projects funded by Shaanxi Natural Science Basic Research Program 
(2019JQ-642), China Postdoctoral Science Foundation (2018M643749), and the

Doctoral Scientific Research Foundation of Northwest A\&F University (Z109021711).

\section{Appendix A. Supplementary data}

Supplementary data to this article can be found online at

\section{References}

Daryanto, S., Wang, L., Jacinthe, P.A., 2015. Global synthesis of drought effects on food legume production. PLoS ONE. 10, e0127401.

Daryanto, S., Wang, L., Jacinthe, P.A., 2016. Global synthesis of drought effects on maize and wheat production. PLoS ONE 11(5): e0156362.

Daryanto, S., Wang, L., Jacinthe, P.A., 2017. Can ridge-furrow plastic mulching replace irrigation in dryland wheat and maize cropping systems? Agric. Water Manage. $190,1-5$

Dong, Q., Yang, Y., Zhang, T., Zhou, L., He, J., Chau, H. W., Zou, Y., Feng, H., 2018 a. Impacts of ridge with plastic mulch-furrow irrigation on soil salinity, spring maize yield and water use efficiency in an arid saline area. Agric. Water Manage. 201, $268-277$.

Dong, W., Yu, H., Wang, R., Wang, Q., Xue, Q., Pan, Z., Sun, Z., Pan, Z., 2018 b. Asymmetric ridge-furrow and film cover improves plant morphological traits and light utilization in rain-fed maize. J. Meteorol. Res. 32, 829-838.

Dong, W., Zhang, L., Duan, Y., Li Sun., Zhao, P., van der Werf, W., Jochem B. Evers, J.B., Wang, Q., Wang, R., Sun, Z., 2017. Ridge and furrow systems with film cover 
increase maize yields and mitigate climate risks of cold and drought stress in continental climates. Field Crop Res. 207, 71-78.

Eldoma, I.M., Li, M., Zhang, F., Li, F., 2016. Alternate or equal ridge-furrow pattern: which is better for maize production in the rain-fed semi-arid loess plateau of China? Field Crop Res. 191, 131-138.

El-Sadek, A., Salem, E., 2015. Impact of ridge-furrow water harvesting system on faba bean (Vicia faba L.) production under rainfed conditions in Matrouh, Egypt. Ann Agric. Sci. 60, 61-66.

Goodman, A.M., Ennos, A.R., 1999. The effects of soil bulk density on the morphology and anchorage mechanics of the root systems of sunflower and maize. Annals of Botany, 83, 293-302.

Hedges, L. V., Olkin, I., 1985. Chapter 5 - estimation of a single effect size: parametric and nonparametric methods. Statistical Methods for Meta-Analysis, 75-106.

Hedges, L.V., Gurevitch, J., Curtis, P.S., 1999. The meta-analysis of response ratios in experimental ecology. Ecology 80, 1150-1156.

Jia, Q., Sun, L., Wang, J., Li, J., Ali, S., Liu, T., Zhang, P., Lian, Y., Ding, R., Ren, X., Jia, Z., 2018a. Limited irrigation and planting densities for enhanced water productivity and economic returns under the ridge-furrow system in semi-arid regions of China. Field Crop Res. 221, 207-218.

Jia, Q., Xua, Y., Ali, S., Sun, L., Ding, R., Ren, X., Zhang, P., Jia, Z., 2018b. Strategies of supplemental irrigation and modified planting densities to improve the root growth and lodging resistance of maize (Zea mays L.) under the ridge-furrow 
rainfall harvesting system. Field Crop Res. 224,48-59.

Li, C., Wang, C., Wen, X., Qin, X., Liu, Y., Han, J., Li, Y., Liao, Y., Wu, Y., 2017a. Ridge-furrow with plastic film mulching practice improves maize productivity and resource use efficiency under the wheat-maize double-cropping system in dry semi-humid areas. Field Crop Res. 203, 201-211.

Li, W., Wen, X., Han, J., Liu, Y., Wu, W., Liao, Y., 2017b. Optimum ridge-to-furrow ratio in ridge-furrow mulching systems for improving water conservation in maize (Zea may L.) production. Environ Sci Pollut Res. 24, 23168-23179.

Li, R., Hou, X.Q., Jia, Z.K., Han, Q.F., Ren, X.L., Yang, B.P., 2013. Effects on soil temperature, moisture, and maize yield of cultivation with ridge and furrow mulching in the rain-fed area of the Loess Plateau, China. Agric. Water Manage. $116,101-109$.

Li, S., Fan, T., Wang, Y., Zhao, G., Wang, L., Tang, X., Dang, Y., Zhao, H., 2014. Effects of plastic film mulching and rain harvesting modes on chlorophyll fluorescence characteristics, yield and water use efficiency of dryland maize. Chin. J. Appl. Ecol. 25, 458-466 (in Chinese).

Li, X., Gong, J., Gao, Q., Li, F., 2001. Incorporation of ridge and furrow method of rainfall harvesting with mulching for crop production under semiarid conditions. Agric. Water Manage. 50, 173-183.

Li, X.L., Su, D.R., Yuan, Q.H., 2007. Ridge-furrow planting of alfalfa (Medicago sativa L.) for improved rainwater harvest in rainfed semiarid areas in Northwest China. Soil Tillage Res. 93, 117-125. 
Liu, X., Li, X., Hai, L., Wang, Y., Li, F., 2014a. How efficient is film fully-mulched ridge-furrow cropping to conserve rainfall in soil at a rainfed site? Field Crop Res. $169,107-115$.

Liu, C.A., Zhou, L.M., Jia, J.J., Wang, L.J., Si, J.T., Li, X., Pan, C.C., Siddique, K.H.M., Li, F.M., 2014b. Maize yield and water balance is affected by nitrogen application in a film-mulching ridge-furrow system in a semiarid region of China. Eur J. Agron. 52, 103-111.

Liu, E.K., He, W.Q., Yan, C.R., 2014c. "White revolution" to "white pollution" Agricultural plastic film mulch in China. Environ. Res. Lett. 9, 091001.

Liu, T., Chen, J., Wang, Z., Wu, X., Wu, X., Ding, R., Han, Q., Cai, T., Jia, Z., 2018. Ridge and furrow planting pattern optimizes canopy structure of summer maize and obtains higher grain yield. Field Crop Res. 219, 242-249.

Mo, F., Wang, J., Xiong, Y., Nguluu, S.N., Li, F., 2016. Ridge-furrow mulching system in semiarid Kenya: a promising solution to improve soil water availability and maize productivity. Eur J. Agron. 80, 124-136.

Moher, D., Liberati, A., Tetzlaff, J., Altman, D.G., The PRISMA Group, 2009. Preferred reporting items for systematic reviews and meta-analyses: the PRISMA statement. PLoS Med. 6, e1000097.

Muhammad, S.M., Zhou, J., Guo, J., Fahim, U., Muhamamd, H., Shamim, A., Ji C., 2017. Comprehensive review for the effects of ridge furrow plastic mulching on crop yield and water use efficiency under different crops. Inter Agric Engine J. 26, 58-67. 
Ng, E. L., Huerta Lwanga, E., Eldridge, S. M., Johnston, P., Hu, H. W., Geissen, V., Chen, D., 2018. An overview of microplastic and nano plastic pollution in agroecosystems. Sci. Total Environ. 627, 1377-1388.

Qin, W., Hu, C., Oenema, O., 2015. Soil mulching significantly enhances yields and water and nitrogen use efficiencies of maize and wheat: A meta-analysis. Sci Rep $5,16210$.

Qin, X., Li, Y., Han, Y., Hu, Y., Li, Y., Wen, X., Liao, Y., Siddique, K.H.M., 2018. Ridge-furrow mulching with black plastic film improves maize yield more than white plastic film in dry areas with adequate accumulated temperature. Agr Forest Meteorol. 262, 206-214.

Ren, X., Chen, X., Cai, T., Wei, T., Wu, Y., Ali, S., Zhang, P., Jia, Z., 2017. Effects of ridge-furrow system combined with different degradable mulching materials on soil water conservation and crop production in semi-humid areas of China. Front. Plant Sci. 8:1877.

Ren, X., Chen, X., Jia, Z., 2010. Effect of rainfall collecting with ridge and furrow on soil moisture and root growth of corn in semiarid northwest China. J. Agron. Crop Sci. 196, 109-122.

Ren, X., Chen, X., Jia, Z., 2016. Impacts of ridge-furrow rainfall concentration systems and mulches on corn growth and yield in the semiarid region of China. J Sci Food Agric. 96, 3882-3889.

Ren, X., Jia, Z., Chen, X., Han, Q., Li, R., 2008. Effects of a rainwater-harvesting furrow/ridge system on spring corn productivity under simulated rainfalls. Acta 
Ecol Sin. 28, 1006-1015.

SAS Institute Inc, 2013. SAS/STAT® 13.1 user's guide. SAS Institute Inc., Cary, NC, USA.

Tian, Y., Zhao, X., Chen, X., Wang, Z., 2015. The influences of fertilization positions on water and nitrate distribution under wheat/maize ridge and furrow intercropping. Agr Res Arid Area. 33, 72-81(in Chinese).

Wang, H., Zhang, X., Song, S., Ma, Y., Yu, X., 2013. Regulation of whole field surface plastic mulching and double ridge-furrow planting on seasonal soil water loss and maize yield in rain-fed area of northwest loess plateau. Sci. Agri. Sin. 46, 917-926 (in Chinese).

Wang, H., Zhang, X., Song, S., Ma, Y., Yu, X., Liu, Y., 2011. Effects of whole fieldsurface plastic mulching and planting in furrow on soil temperature, soil moisture, and corn yield in arid area of Gansu Province, Northwest China. Chin. J. Appl. Ecol. 22, 2609-2614(in Chinese).

Wang, X., Chen, M., Yi, X., Fu, G., 2009. Effects of ridge width and planting density on corn yields in rainwater-harvesting system with plastic film mulching on ridge. Transactions of the CSAE. 25, 40-47(in Chinese).

Wang, Y., Gao, F., Yang, J., Zhao, J., Wang, X., Gao, G., Zhang, R., Jia, Z., 2018a. Spatio-temporal variation in dryland wheat yield in northern Chinese areas: relationship with precipitation, temperature and evapotranspiration. Sustainability $10,4470$.

Wang, H., Zhang, X., Yu, X., Hou, H., Fang, Y., Ma, Y., 2018b. Maize-faba bean 
rotation under double ridge and furrows with plastic mulching alleviates soil water depletion. Agric. Water Manage. 207, 59-66.

Wang, Y., Zhang, Y., Zhou, S., Wang, Z., 2018c. Meta-analysis of no-tillage effect on wheat and maize water use efficiency in China. Sci. Total Environ. 635, 13721382.

Wu, J., Xiao, K., Zhao, C., Yu, A., Feng, F., Li, L., Chai, Q., 2018. Ridge-furrow cropping of maize reduces soil carbon emissions and enhances carbon use efficiency. Agr. Ecosyst. Environ. 256, 153-162.

Wu, Y., Jia, Z., Ren, X., Zhang, Y., Chen, X., Bing, H., Zhang, P., 2015. Effects of ridge and furrow rainwater harvesting system combined with irrigation on improving water use efficiency of maize (Zea mays L.) in semi-humid area of China. Agric. Water Manage. 158, 1-9.

Yin, M., Li, Y., Xu, Y., Zhou, C., 2018. Effects of mulches on water use in a winter wheat/summer maize rotation system in Loess Plateau, China. J Arid Land 10, $277-291$.

Yu, Y. Y., Turner, N. C., Gong, Y. H., Li, F. M., Fang, C., Ge, L. J., Ye, J. S., 2018. Benefits and limitations to straw- and plastic-film mulch on maize yield and water use efficiency: A meta-analysis across hydrothermal gradients. Eur J. Agron. 99, $138-147$.

Yu, Y., Stomph, T. J., Makowski, D., van der Werf, W., 2015. Temporal niche differentiation increases the land equivalent ratio of annual intercrops: A metaanalysis. Field Crop Res 184, 133-144. 
Zhang, S. L., Li, P. R., Yang, X.Y., Wang, Z. H., Chen, X. P., 2011. Effects of tillage and plastic mulch on soil water, growth and yield of spring-sown maize. Soil Till Res. 112, 92-97.

Zhang, Y., Ma, Q., Liu, D., Sun, L., Ren, X., Ali, S., Zhang, P., Jia, Z., 2018. Effects of different fertilizer strategies on soil water utilization and maize yield in the ridge and furrow rainfall harvesting system in semiarid regions of China. Agric. Water Manage. 208, 414-421.

Zheng, J., Fan, J., Zhang, F., Yan, S., Guo, J., Chen, D., Li, Z., 2018. Mulching mode and planting density affect canopy interception loss of rainfall and water use efficiency of dryland maize on the Loess Plateau of China. J. Arid Land 10, 794808.

Zhou, L., Jin, S., Liu, C., Xiong, Y., Si, J., Li, X., Gan, Y., Li, F., 2012. Ridge-furrow and plastic-mulching tillage enhances maize-soil interactions: opportunities and challenges in a semiarid agroecosystem. Field Crop Res. 126, 181-188.

Zhou, L., Li, F., Jin, S., Song, Y., 2009. How two ridges and the furrow mulched with plastic film affect soil water, soil temperature and yield of maize on the semiarid loess plateau of China. Field Crop Res. 113, 41-47. 


\section{Figure 1}

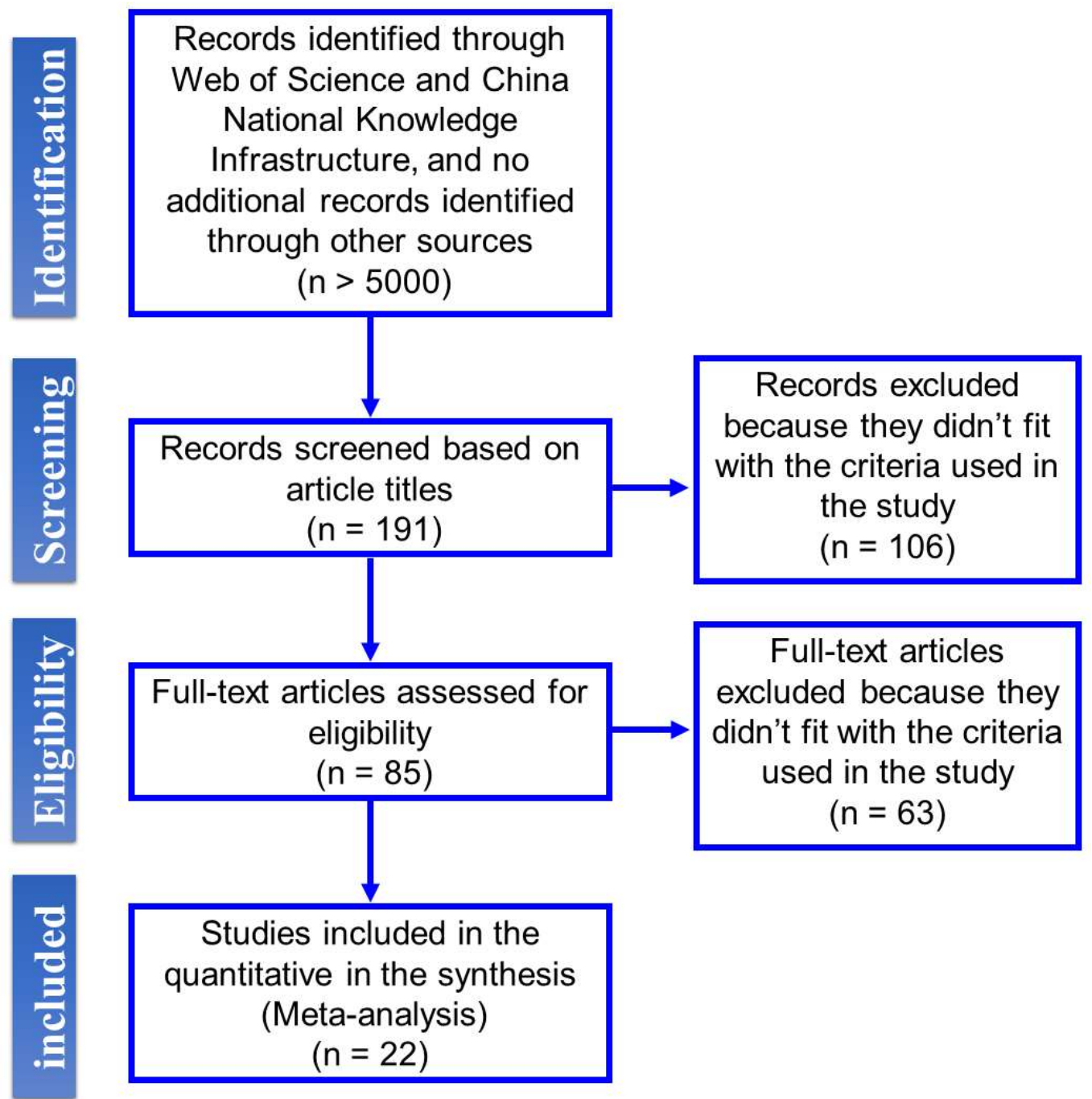

Fig. 1. Flowchart of the process used to obtain the literature data to build a database for this study. 


\section{Figure 2}

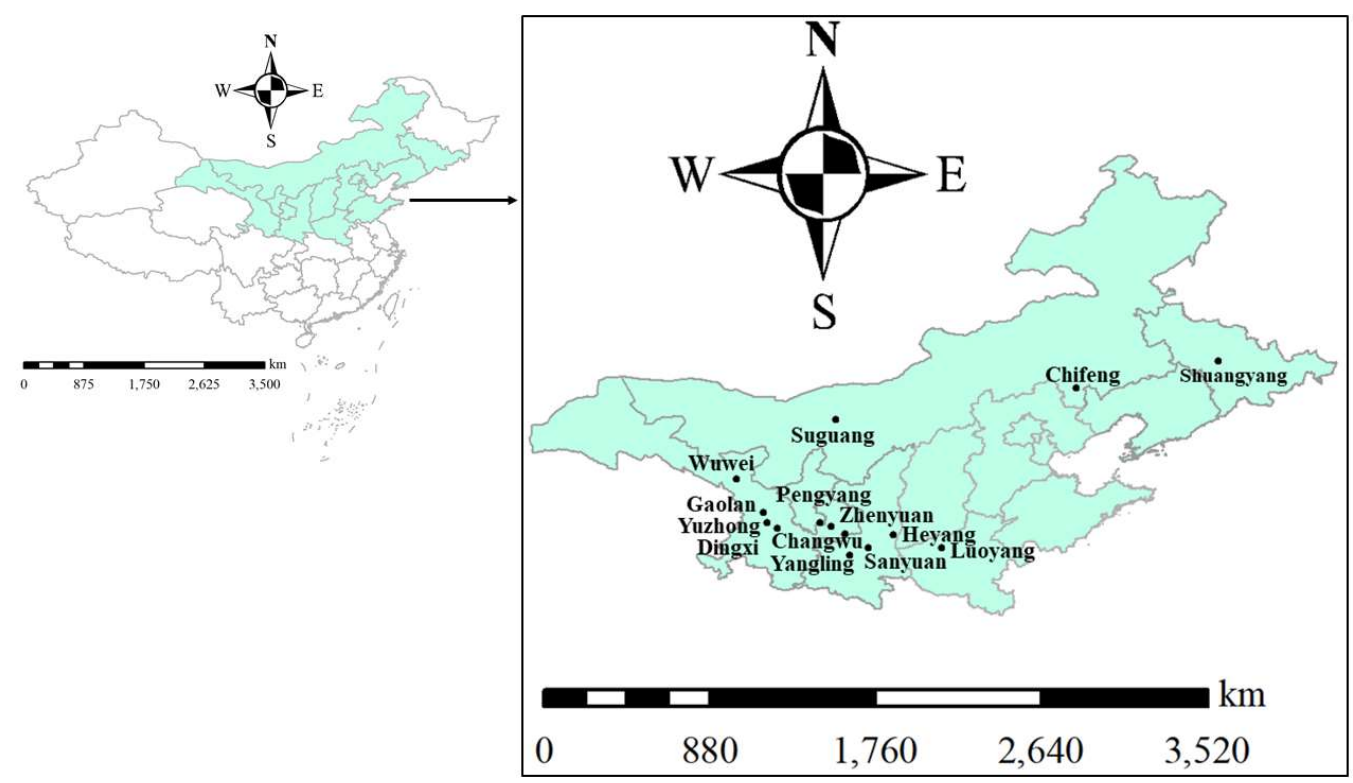

Fig. 2. Distribution of the locations of the studies included in the meta-analysis. The map was generated using ArcGIS 10.2. 


\section{Figure 3}

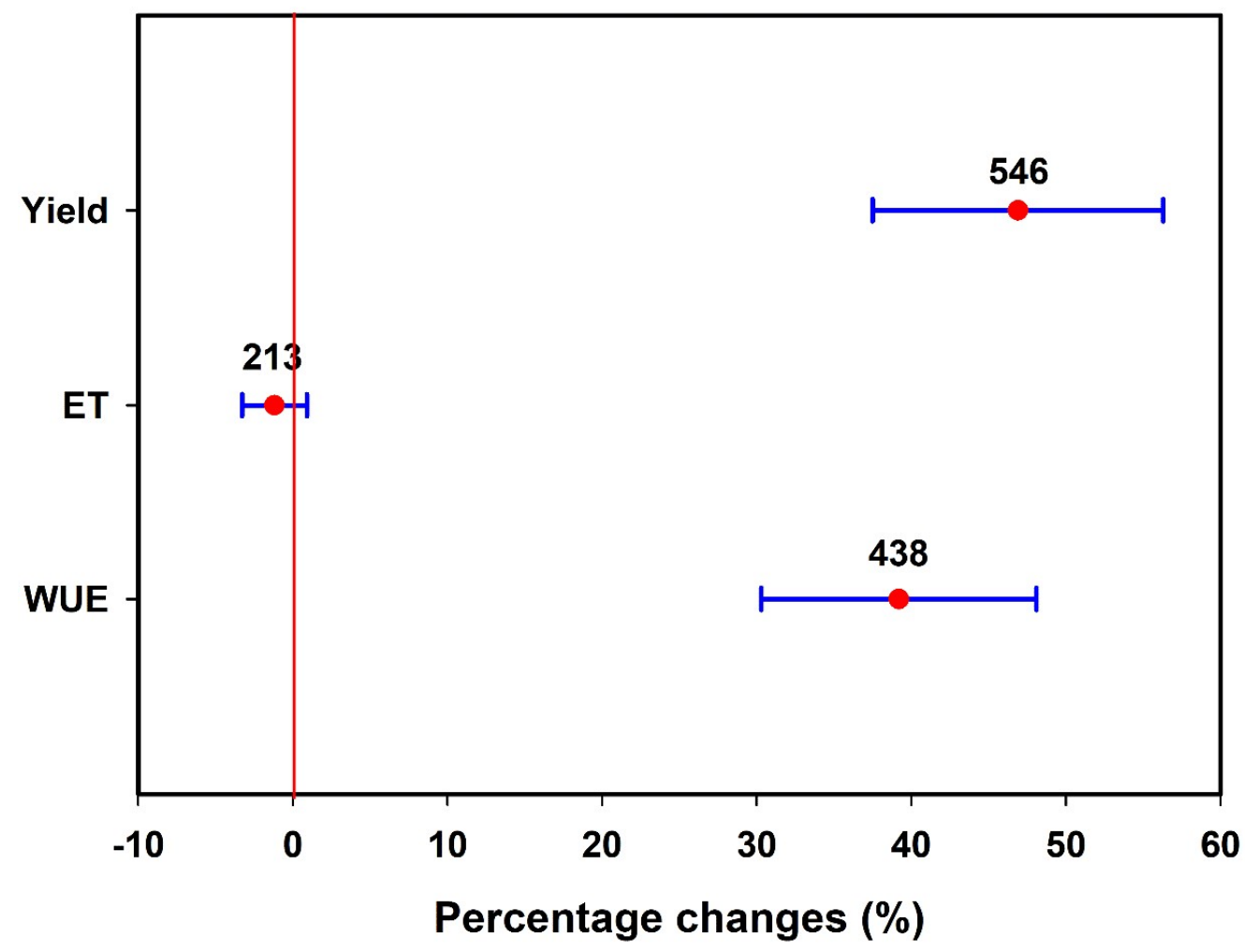

Fig. 3. Percentage changes in maize yield, ET and WUE comparing ridge-furrow cultivation to flat-plot cultivation across a wide range of environmental and management conditions. Error bars are the $95 \%$ confidence intervals (CIs). The number of observations is indicated over the CIs. The 213 WUE observations were calculated through WUE $=$ Yield/ET, and 225 WUE observations were directly from references. 


\section{Figure 4}
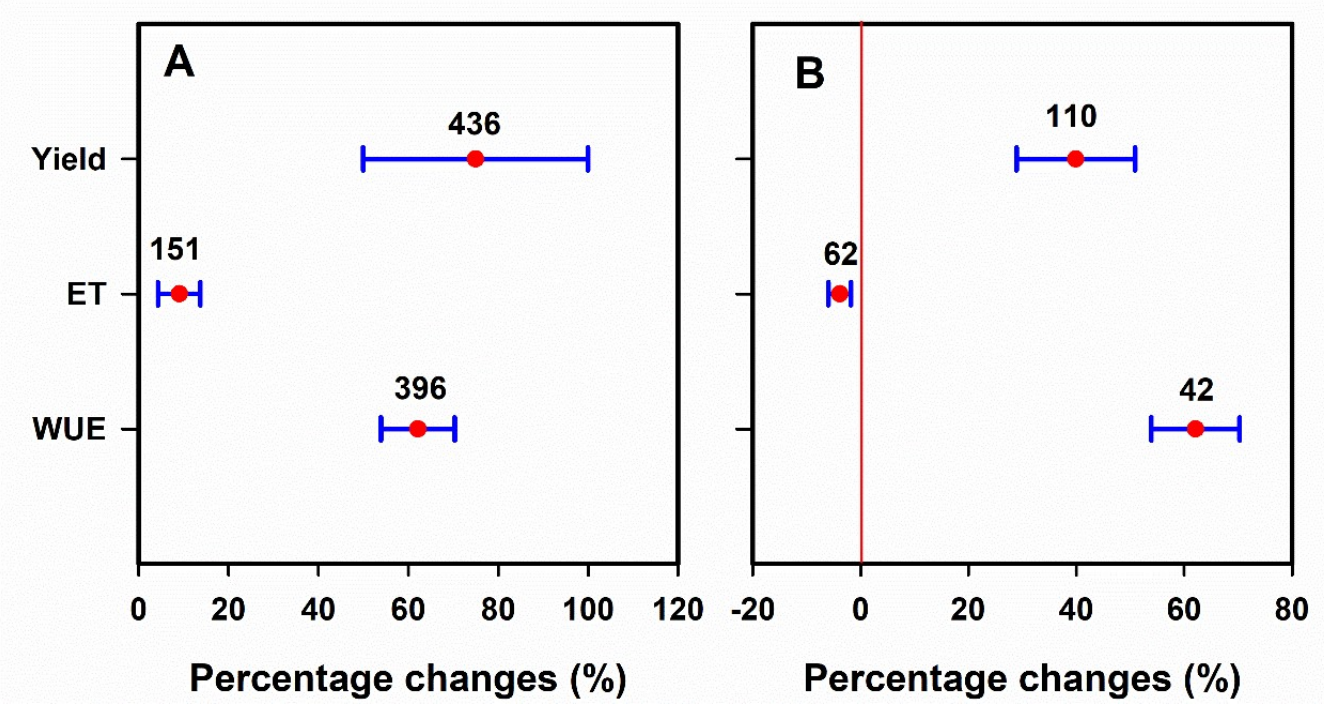

Fig. 4. Percentage changes in maize yield, ET and WUE comparing ridge-furrow cultivation to flat-plot cultivation under different mean growing season air temperature of maize $\left(\mathrm{A}, \leq 12{ }^{\circ} \mathrm{C} ; \mathrm{B},>12{ }^{\circ} \mathrm{C}\right)$. Error bars are the $95 \%$ confidence intervals (CIs). The number of observations is indicated over the CIs. The 213 WUE observations were calculated through WUE $=$ Yield/ET, and 183 WUE observations were directly from references in Fig. 4A. All the WUE observations were directly from references in Fig. 4B. 


\section{Figure 5}
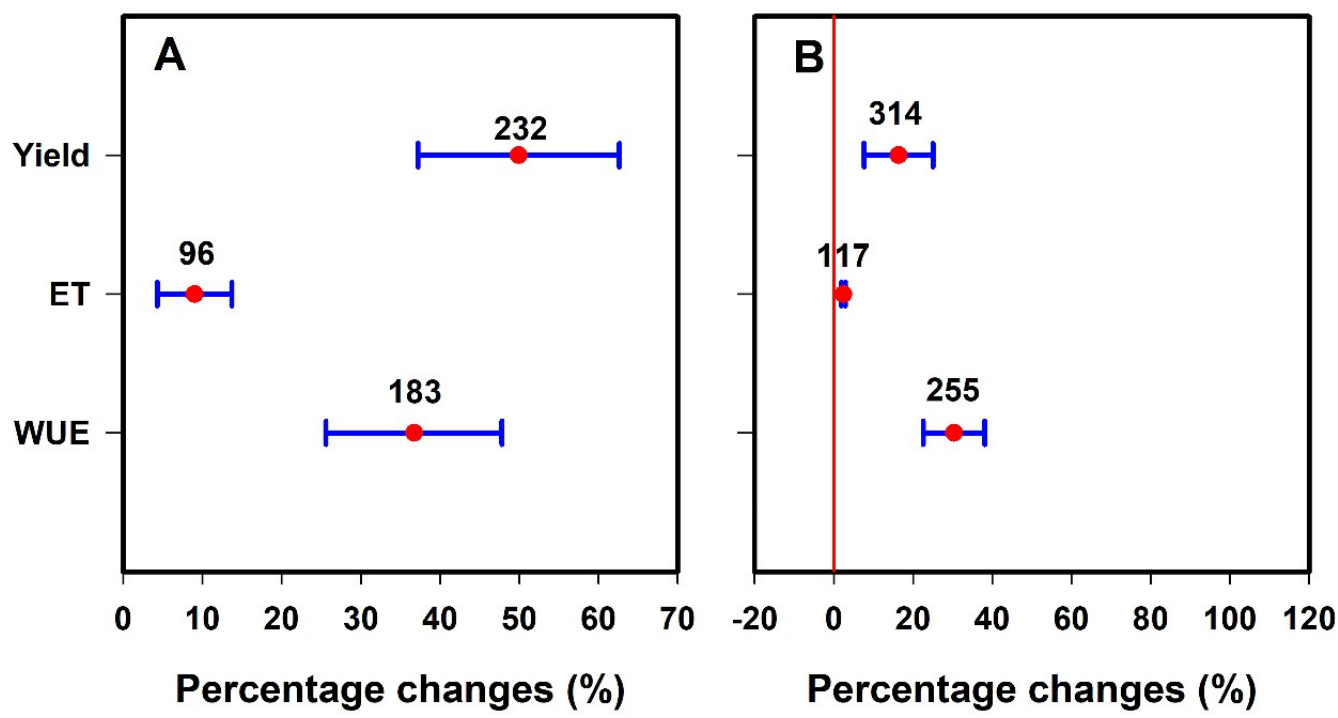

Fig 5. Percentage changes in maize yield, ET and WUE comparing ridge-furrow cultivation to flat-plot cultivation under different mean precipitation during the growing season of maize $(\mathrm{A}, \leq 400 \mathrm{~mm} ; \mathrm{B},>400 \mathrm{~mm})$. Error bars are the $95 \%$ confidence intervals (CIs). The number of observations is indicated over the CIs. The 96 WUE observations were calculated through WUE $=$ Yield/ET, and 87 WUE observations were directly from references in Fig. 5A. The 117 WUE observations was calculated through WUE $=$ Yield/ET, and 138 WUE observations were directly from references in Fig. 5B. 


\section{Figure 6}
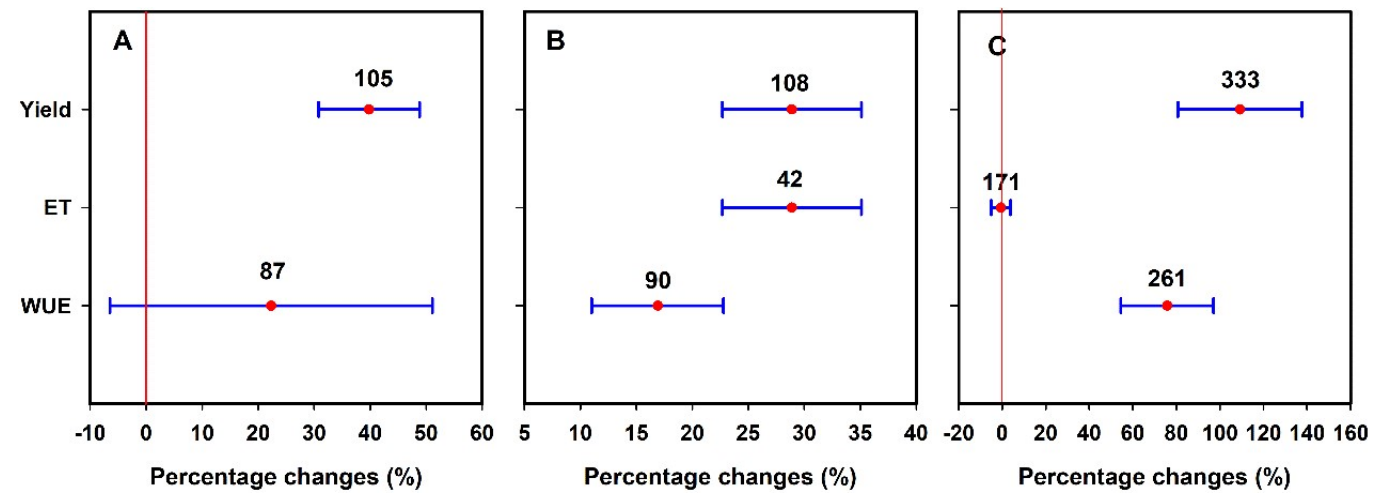

Fig. 6. Percentage changes in maize yield, ET and WUE comparing ridge-furrow cultivation to flat-plot cultivation under different soil textures (A, coarse; B, medium; C, fine). Error bars are the $95 \%$ confidence intervals (CIs). The number of observations is indicated over the CIs. All the WUE observations were directly from references in Fig. $6 \mathrm{~A}$. The $42 \mathrm{WUE}$ observations were calculated through WUE $=$ Yield/ET, and 48 WUE observations were directly from references in Fig. 6B. The 171 WUE observations were calculated through WUE $=$ Yield/ET, and $90 \mathrm{WUE}$ observations were directly from references in Fig. 6C. 
Figure 7
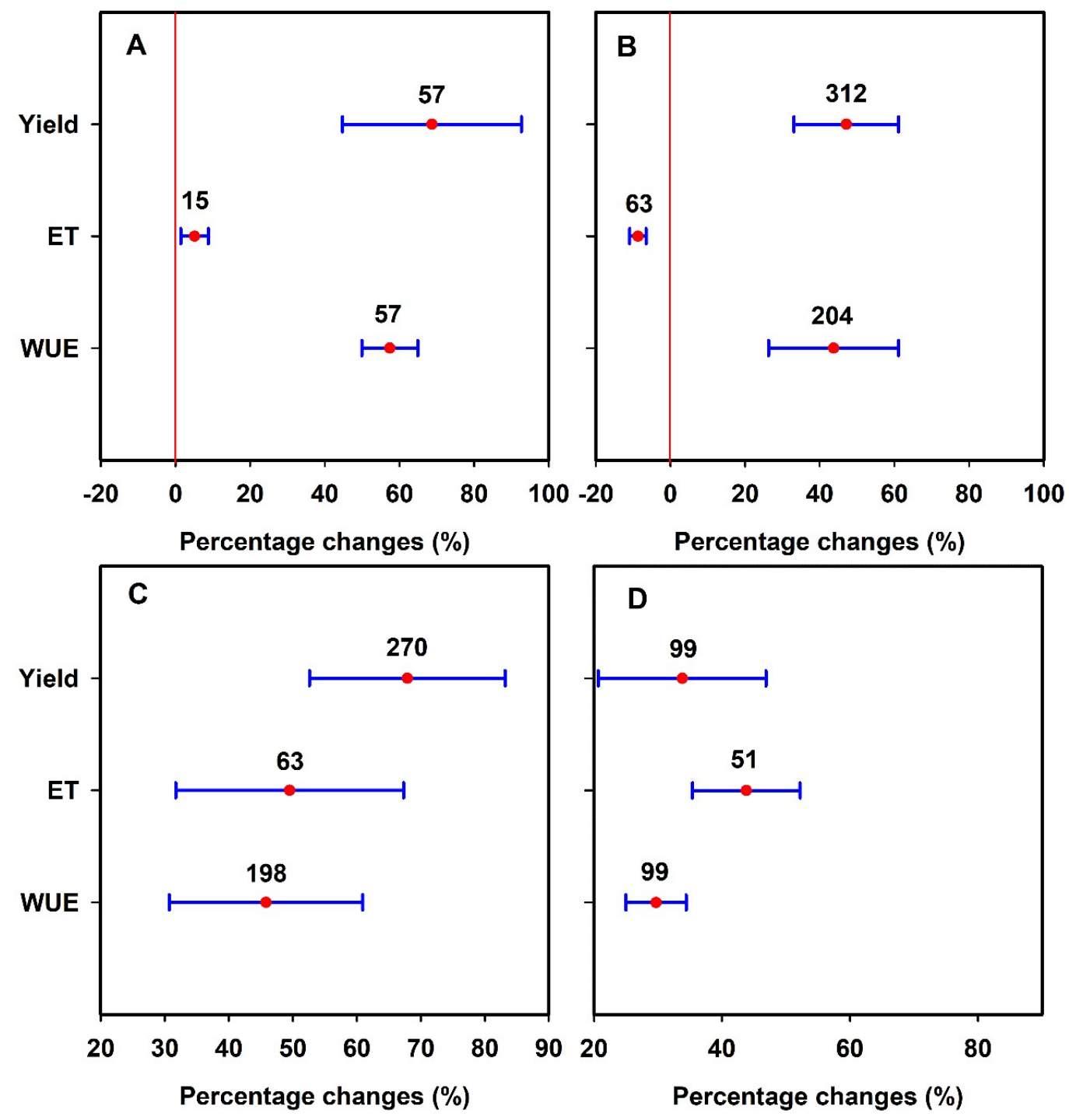

Fig. 7. Percentage changes in maize yield, ET and WUE comparing ridge-furrow cultivation to flat-plot cultivation under different soil bulk density $\left(A, \leq 1.3 \mathrm{~g} \mathrm{~cm}^{-3}\right.$; $\left.\mathrm{B},>1.3 \mathrm{~g} \mathrm{~cm}^{-3}\right)$, and with different field capacity $(\mathrm{C}, \leq 25 \%$;,$>25 \%)$. Error bars are the $95 \%$ confidence intervals (CIs). The number of observations is indicated over the CIs. The 15 WUE observations were calculated through WUE $=$ Yield/ET, and 42 WUE observations were directly from references in Fig. 7A. The 63 WUE observations were calculated through WUE $=$ Yield/ET, and 141 WUE observations were directly from 
references in Fig. 7B. The 63 WUE observations were calculated through WUE $=$ Yield/ET, and 135 WUE observations were directly from references in Fig. 7C. The 51 WUE observations were calculated through WUE $=$ Yield/ET, and $48 \mathrm{WUE}$ observations were directly from references in Fig. 7D. 


\section{Figure 8}
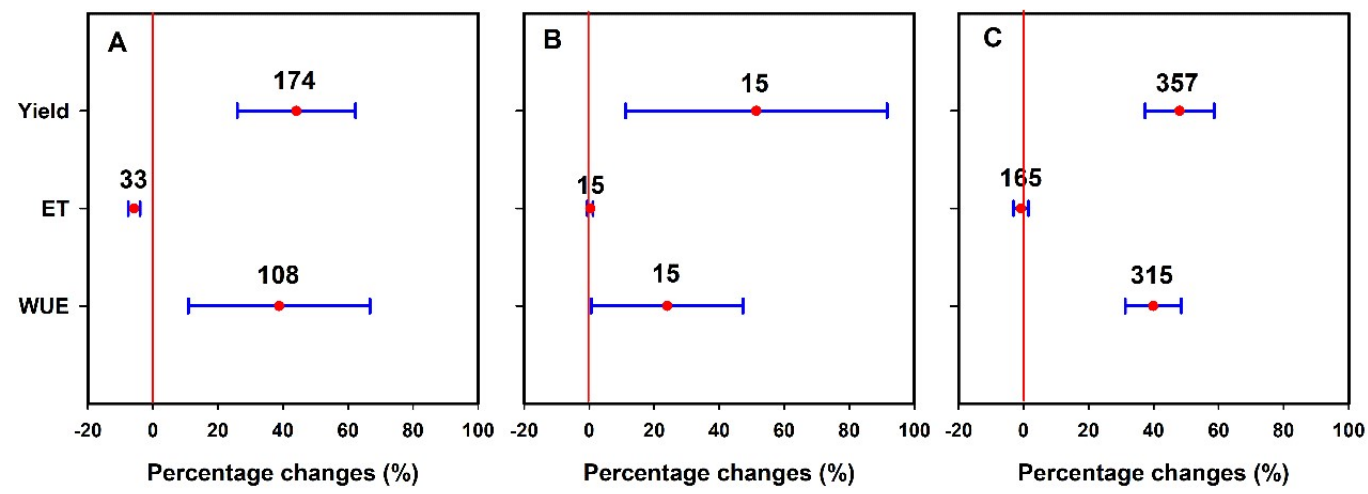

Fig. 8. Percentage changes in maize yield, ET and WUE comparing ridge-furrow cultivation to flat-plot cultivation under different mulching management (A, ridgefurrow; B, ridge-furrow mulched with straw; $\mathrm{C}$, ridge-furrow mulched with plastic film). Error bars are the $95 \%$ confidence intervals (CIs). The number of observations is indicated over the CIs. The 33 WUE observations were calculated through WUE $=$ Yield/ET, and 75 WUE observations were directly from references in Fig. 8A. All the WUE observations were calculated through WUE $=$ Yield/ET in Fig. 8B. The $165 \mathrm{WUE}$ observations were calculated through WUE $=$ Yield/ET, and 150 WUE observations were directly from references in Fig. 8C. 


\section{Table 1}

Characteristics of the studies used in the meta-analysis.

\begin{tabular}{|c|c|c|c|c|c|c|c|c|c|c|}
\hline Reference & Location & $\operatorname{MT}\left({ }^{\circ} \mathrm{C}\right)$ & $\mathrm{MP}(\mathrm{mm})$ & ST & $\mathrm{BD}\left(\mathrm{g} \mathrm{cm}^{-}\right.$ & $\mathrm{FC}(\%)$ & MM & $\mathrm{NC}$ of & $\mathrm{NC}$ of ET & $\mathrm{NC}$ of \\
\hline Dong et al. (2017) & Chifeng, Inner & 18.3 & 331.0 & Medium & 1.37 & 1 & RF, RFP & 9 & 0 & 0 \\
\hline Dong et al. (2017) & Shuangyang, Jilin, & 17.6 & 477.5 & Medium & 1.43 & 1 & RF, RFP & 18 & 0 & 0 \\
\hline Dong et al. (2018a) & Suguang, Inner & 20.9 & 136.5 & Medium & 1.33 & 1 & RF, RFP & 9 & 0 & 0 \\
\hline Dong et al. (2018b) & Shuangyang, Jilin, & 17.6 & 477.5 & Medium & 1.43 & 1 & RFP & 36 & 36 & 36 \\
\hline Eldoma et al. (2016) & Yuzhong, Gansu, & 18.8 & 269.0 & Fine & 1.32 & 22.9 & $\mathrm{RF}, \mathrm{RFP}$ & 24 & 0 & 24 \\
\hline Li et al. (2001) & Gaolan, Gansu, & 17.1 & 226.0 & Coarse & 1.35 & 22.9 & RF, RFS, & 42 & 0 & 42 \\
\hline Li et al. (2014) & Zhenyuan, Gansu, & 16.6 & 344.5 & Medium & 1 & 1 & RFP & 63 & 63 & 63 \\
\hline Li et al. (2017a) & Changwu, Shaanxi, & 11.8 & 262.0 & Medium & 1.30 & 26.6 & RFP & 18 & 0 & 18 \\
\hline Li et al. (2017b) & Sanyuan, Shaanxi, & 20.9 & 506.0 & Medium & 1.32 & 26.9 & RFP & 18 & 0 & 18 \\
\hline Liu et al. (2018) & Yangling, Shaanxi, & 21.2 & 537.1 & Coarse & 1.37 & 22.0 & $\mathrm{RF}$ & 18 & 0 & 0 \\
\hline Qin et al. (2018) & Changwu, Shaanxi, & 11.8 & 262.0 & Fine & 1.30 & 26.6 & RFP & 12 & 0 & 12 \\
\hline Ren et al. (2008) & Yangling, Shaanxi, & 21.2 & 537.1 & Medium & 1.25 & 23.0 & RFP & 9 & 9 & 9 \\
\hline Ren et al. (2010) & Yangling, Shaanxi, & 21.2 & 537.1 & Medium & 1.35 & 22.0 & $\mathrm{RF}$ & 18 & 0 & 0 \\
\hline Ren et al. (2016) & Pengyang, Ningxia, & 14.9 & 391.0 & Coarse & 1.39 & 21.0 & RF, RFP & 45 & 0 & 45 \\
\hline Ren et al. (2017) & Heyang, Shaanxi, & 13.8 & 378.8 & Medium & 1.34 & 27.8 & RF, RFP & 45 & 45 & 45 \\
\hline Tian et al. (2015) & Yangling, Shaanxi, & 21.2 & 537.1 & Medium & 1.37 & 22.0 & $\mathrm{RF}$ & 30 & 0 & 12 \\
\hline Wang et al. (2009) & Luoyang, Henan, & 15.6 & 350.0 & Fine & 1 & 1 & RFP & 18 & 18 & 18 \\
\hline Wang et al. (2011) & Dingxi, Gansu, & 11.4 & 253.3 & Fine & 1.25 & 23.2 & RF, RFP & 12 & 0 & 12 \\
\hline Wang et al. (2013) & Dingxi, Gansu, & 11.4 & 253.3 & Fine & 1.25 & 26.0 & RFP & 6 & 6 & 6 \\
\hline Wu et al. (2018) & Wuwei, Gansu, & 17.3 & 140.6 & Medium & 1 & 1 & RFP & 42 & 0 & 42 \\
\hline Zheng et al. (2018) & Yangling, Shaanxi, & 21.2 & 537.1 & Medium & 1.25 & 23.0 & RFS, RFP & 18 & 18 & 18 \\
\hline Zhou et al. (2009) & Yuzhong, Gansu, & 18.8 & 269.0 & Fine & 1.35 & 22.9 & $\mathrm{RF}$ & 18 & 18 & 18 \\
\hline Zhou et al. (2012) & Yuzhong, Gansu, & 18.8 & 269.0 & Fine & 1.35 & 22.9 & RFP & 18 & 0 & 0 \\
\hline
\end{tabular}

MT: mean growing season air temperature of maize from each reference; MP: mean

precipitation during the growing season of maize from each reference; BD: soil bulk density from each reference; FC: field capacity from each reference; MM: mulching management from each reference; RF: ridge-furrow; RFS: ridge-furrow mulched with straw; RFP: ridge-furrow mulched with plastic film; NC: number of comparisons. 


\section{Table 2}

Correlation coefficients between the $\ln R$ values of WUE with those of yield and ET under different environmental and management factors.

\begin{tabular}{llll}
\hline Factor & & Yield & ET \\
\hline Grand & & $0.86^{* * * *}$ & $-0.36^{*}$ \\
MT & $\leq 12{ }^{\circ} \mathrm{C}$ & $0.89^{* * * *}$ & $-0.61^{* *}$ \\
& $>12{ }^{\circ} \mathrm{C}$ & $0.97^{* * * *}$ & $-0.58^{*}$ \\
MP & $\leq 400 \mathrm{~mm}$ & $0.79^{* *}$ & $-0.61^{* *}$ \\
& $>400 \mathrm{~mm}$ & $0.92^{* * * *}$ & $-0.82^{* *}$ \\
Soil texture & Coarse & $0.48^{* *}$ & $\backslash$ \\
& Medium & $0.51^{* * * *}$ & $-0.06^{\mathrm{ns}}$ \\
\multirow{3}{*}{ Soil bulk density } & Fine & $0.93^{* * * *}$ & $-0.17^{\mathrm{ns}}$ \\
& $\leq 1.3 \mathrm{~g} \mathrm{~cm}{ }^{-3}$ & $0.99^{* * * *}$ & $0.81^{*}$ \\
Field capacity & $>1.3 \mathrm{~g} \mathrm{~cm}{ }^{-3}$ & $0.44^{*}$ & $-0.05^{\mathrm{ns}}$ \\
& $<25 \%$ & $0.89^{* * * *}$ & $-0.63^{* *}$ \\
Mulching management & $\geq 25 \%$ & $0.24^{* * * *}$ & $0.17^{\mathrm{ns}}$ \\
& Ridge-furrow & $0.90^{* * * *}$ & $-0.37^{\mathrm{ns}}$ \\
& Ridge-furrow mulched with straw & $0.67^{* * * *}$ & $0.50^{\mathrm{ns}}$ \\
& Ridge-furrow mulched with plastic film & $0.45^{* * *}$ & $-0.01^{\mathrm{ns}}$ \\
\hline
\end{tabular}

ET: evapotranspiration; MT: mean growing season air temperature of maize; MP: mean precipitation during the growing season of maize; ns: not significant. ${ }^{*}, * *, * * * *$ and $* * * *$ indicate significance at $P<0.05, P<0.01, P<0.001$ and $P<0.0001$, respectively. 


\section{Table 3}

Correlation coefficients between possible influential factors (MP, MT, BD and FC) and the $\ln R$ of yield, ET and WUE.

\begin{tabular}{llll}
\hline Factor & Yield & ET & WUE \\
\hline MT & $-0.24^{*}$ & $0.79^{* *}$ & $0.16^{\text {ns }}$ \\
MP & $-0.39^{*}$ & $0.91^{* *}$ & $0.09^{\text {ns }}$ \\
BD & $-0.28^{* *}$ & $-0.78^{* * *}$ & $-0.18^{\text {ns }}$ \\
FC & $-0.14^{\text {ns }}$ & $-0.38^{*}$ & $-0.05^{\text {ns }}$ \\
\hline
\end{tabular}

ET: evapotranspiration; MT: mean growing season air temperature of maize; MP: mean precipitation during the growing season of maize; BD: soil bulk density; FC: field capacity; ns: not significant. *, ** and **** indicate significance at $P<0.05, P<0.01$ and $P<0.0001$, respectively. 


\section{Supplementary Information.}
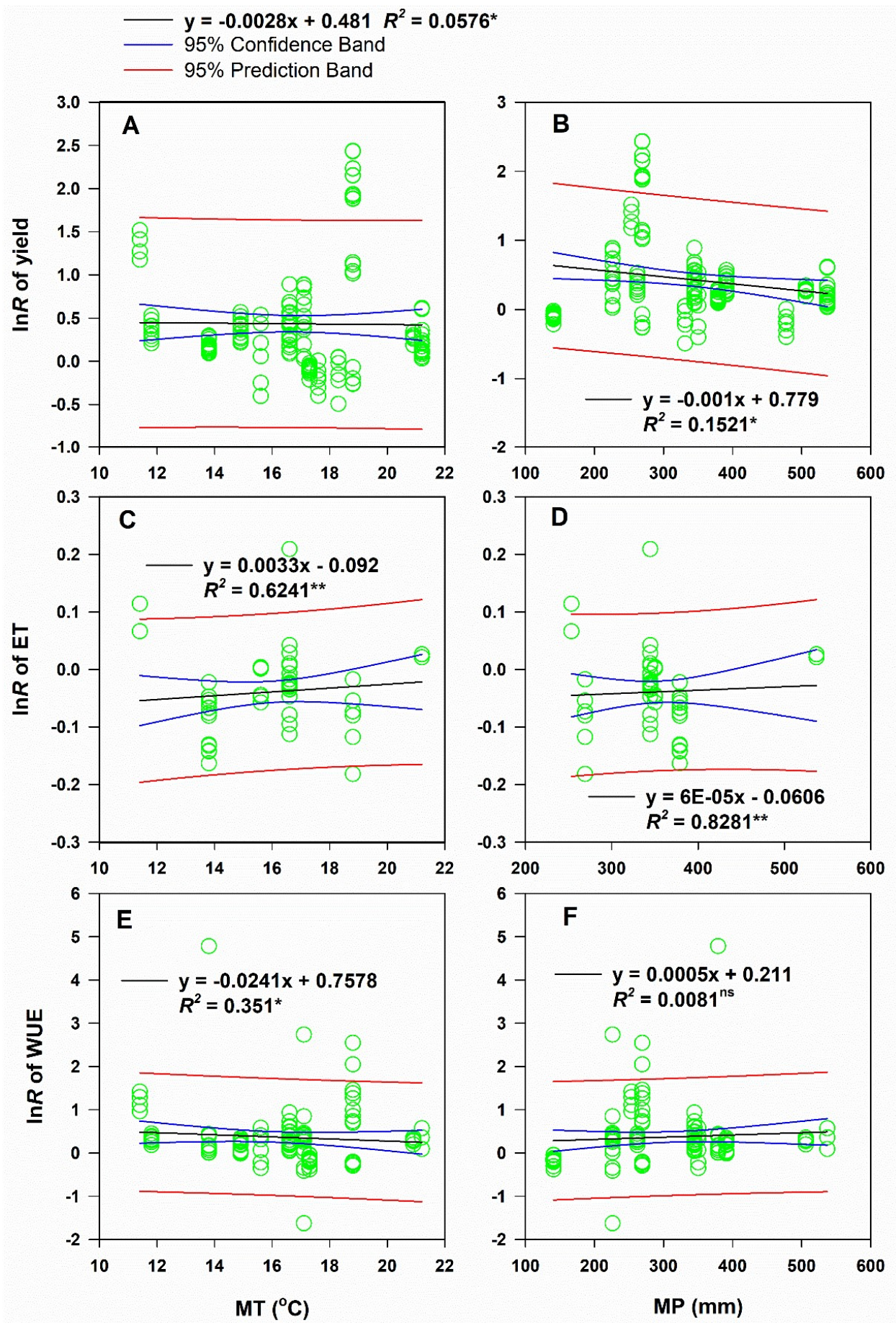

Fig. S1. Scatter plots and linear models relating $\ln R$ to $\mathrm{MT}(\mathrm{A}, \mathrm{C}, \mathrm{E})$ and $\mathrm{MP}(\mathrm{B}, \mathrm{D}, \mathrm{F})$. Relationship between $\ln R$ and MT and MP were estimated by a mixed effects model: $\ln R=\beta 0+\beta 1 * \mathrm{MT}+\beta 2, \ln R=\beta 0+\beta 1 * \mathrm{MP}+\beta 2$. ns: not significant, ${ }^{*} \mathrm{P}<0.05,{ }^{* *} \mathrm{P}<$ 0.01. MT: mean air temperature during the growth period of maize; MP: mean precipitation during the growth period of maize. 

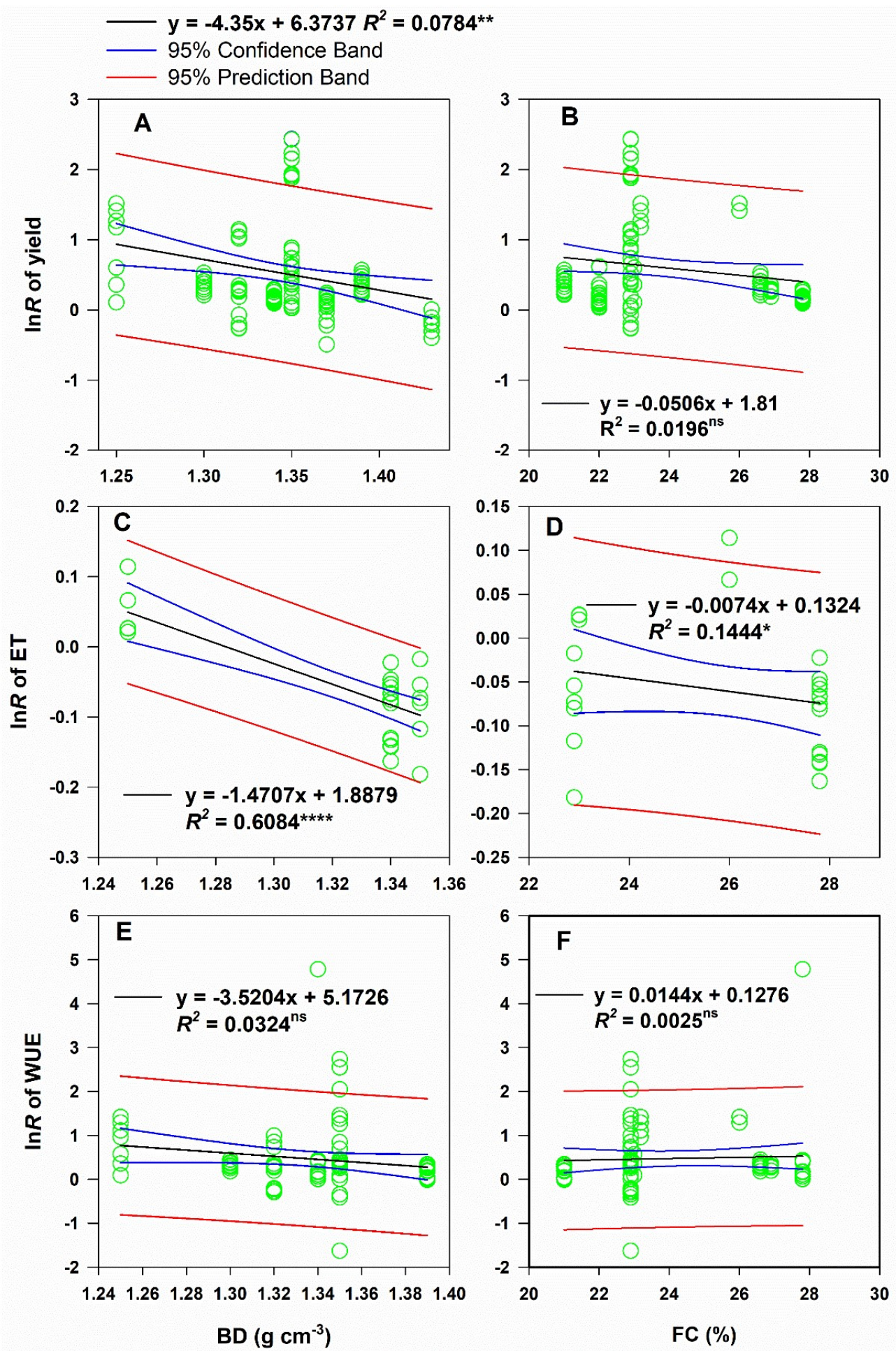

Fig. S2. Scatter plots and linear models relating $\ln R$ to $\mathrm{BD}(\mathrm{A}, \mathrm{C}, \mathrm{E})$ and $\mathrm{FC}(\mathrm{B}, \mathrm{D}, \mathrm{F})$. Relationship between $\ln R$ and MT and MP were estimated by a mixed effects model: $\ln R=\beta 0+\beta 1 * \mathrm{BD}+\beta 2, \ln R=\beta 0+\beta 1 * \mathrm{FC}+\beta 2$. ns: not significant, ${ }^{*} \mathrm{P}<0.05,{ }^{* *} \mathrm{P}<$ $0.01,{ }^{* * * *} \mathrm{P}<0.0001$. BD: soil bulk density; FC: field capacity. 


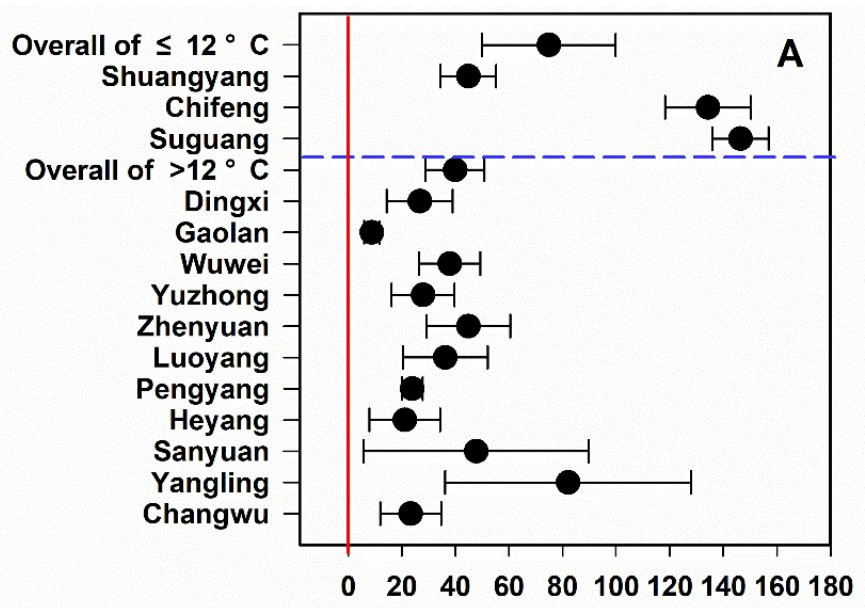

Percentage changes of yield (\%)

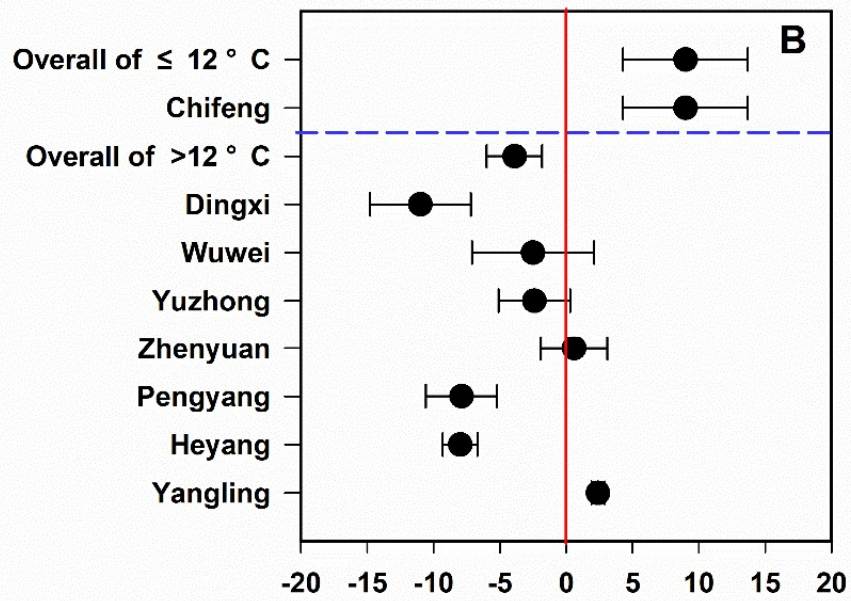

Percentage changes of ET (\%)

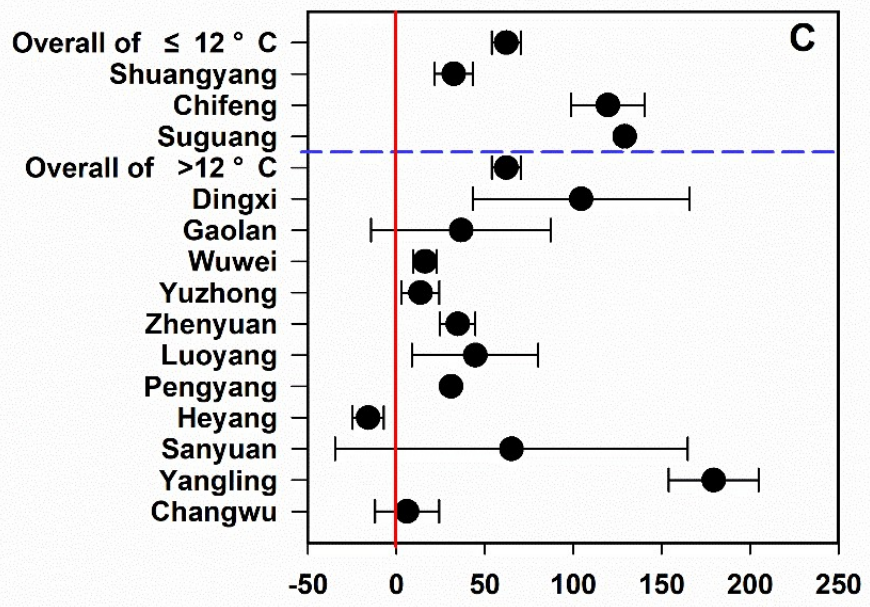

\section{Percentage changes of WUE (\%)}

Fig. S3. Percentage changes in maize yield (A), ET (B) and WUE (C) comparing ridgefurrow cultivation to flat-plot cultivation under different mean air temperature during the growth period. Error bars are the 95\% confidence intervals (CIs). 


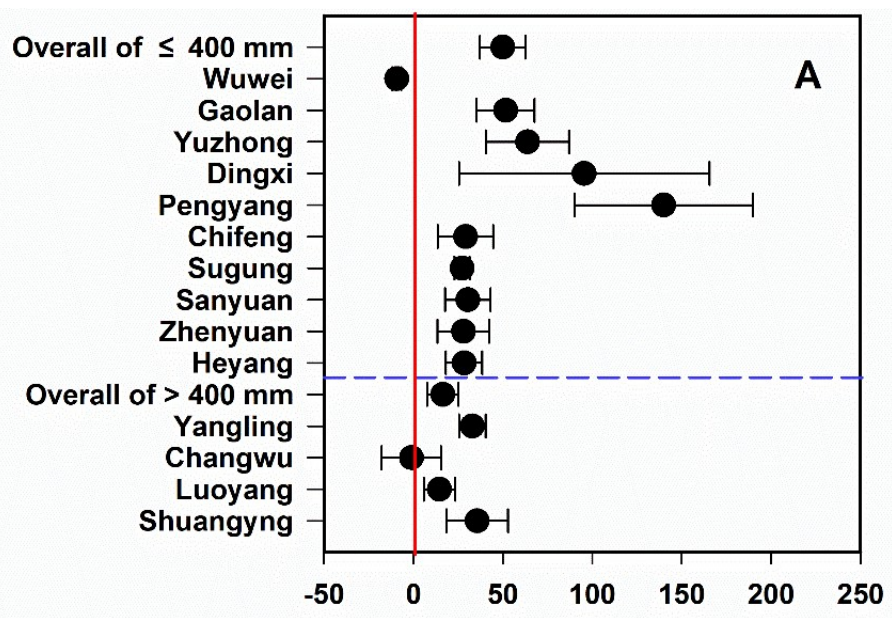

Percentage changes of yield (\%)
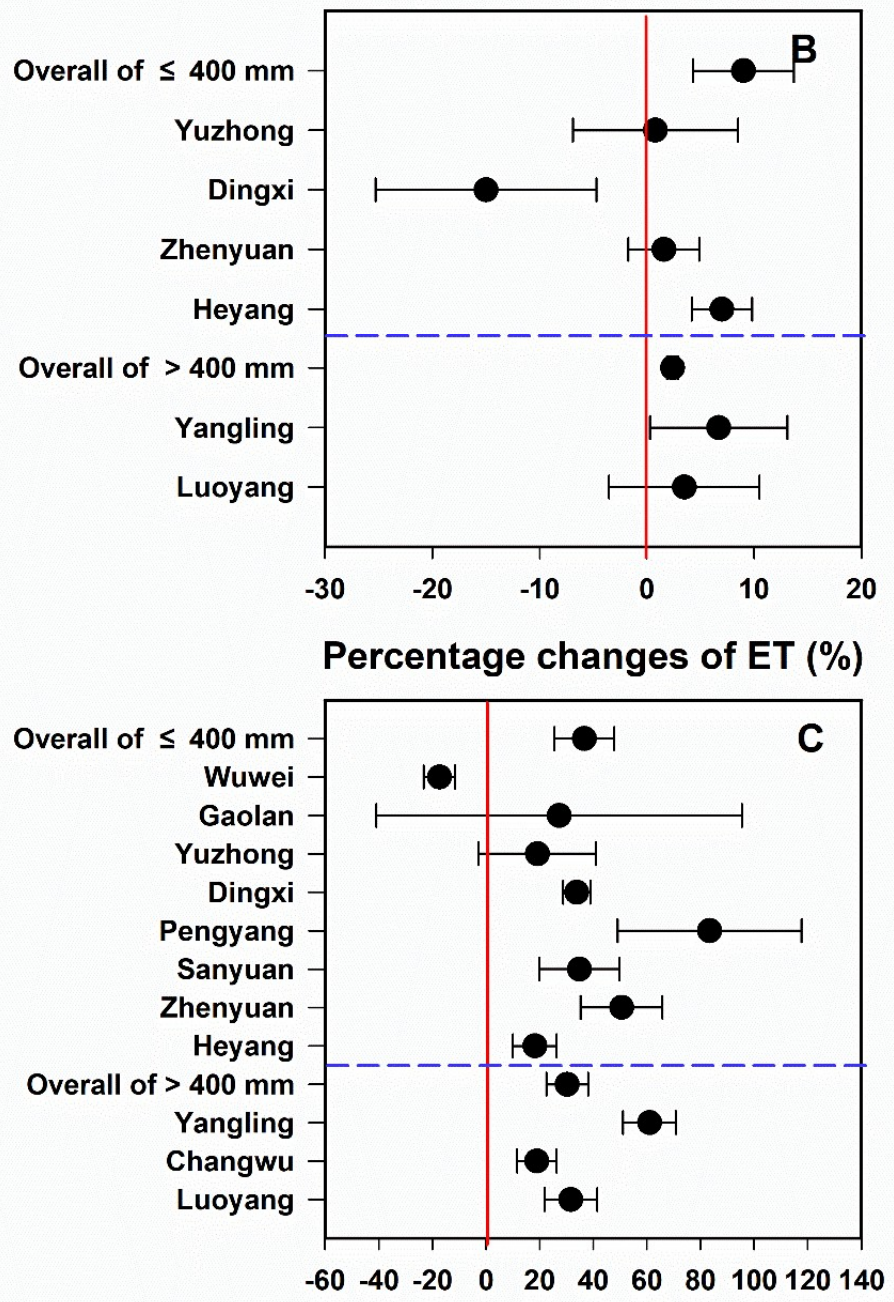

Percentage changes of WUE (\%)

Fig. S4. Percentage changes in maize yield (A), ET (B) and WUE (C) comparing ridgefurrow cultivation to flat-plot cultivation under different mean precipitation during the growth period. Error bars are the $95 \%$ confidence intervals (CIs). 


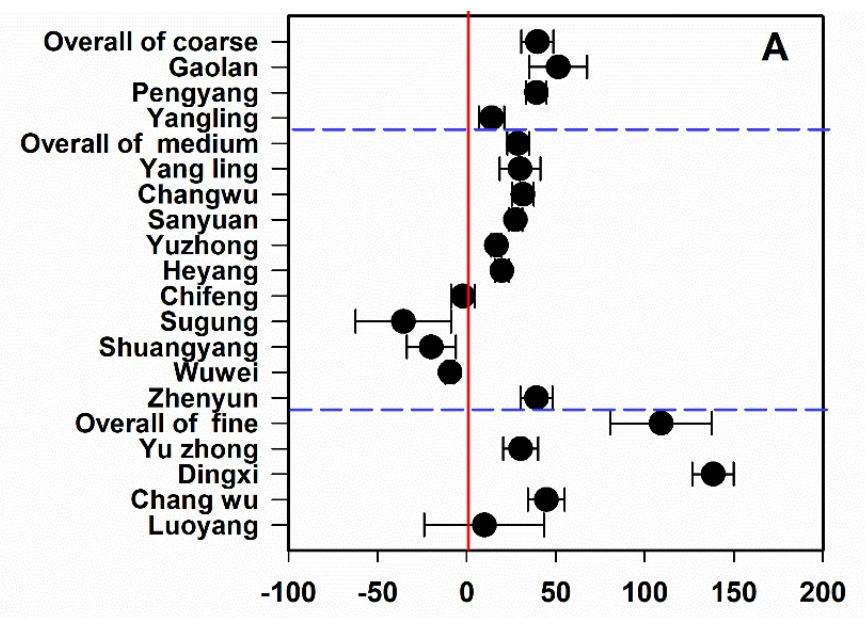

Percentage changes of yield (\%)

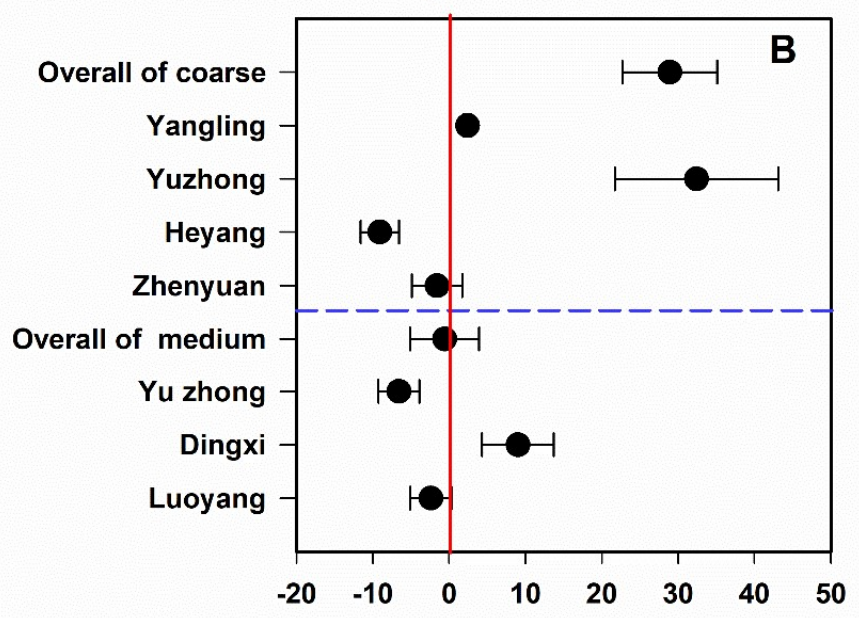

Percentage changes of ET (\%)

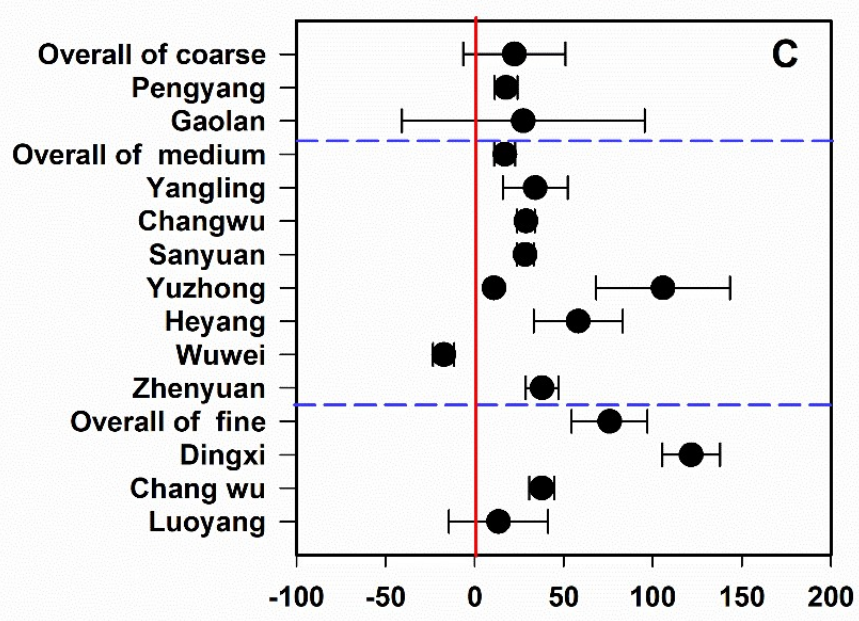

Percentage changes of WUE (\%)

Fig. S5. Percentage changes in maize yield (A), ET (B) and WUE (C) comparing ridgefurrow cultivation to flat-plot cultivation under different soil textures. Error bars are the 95\% confidence intervals (CIs). 


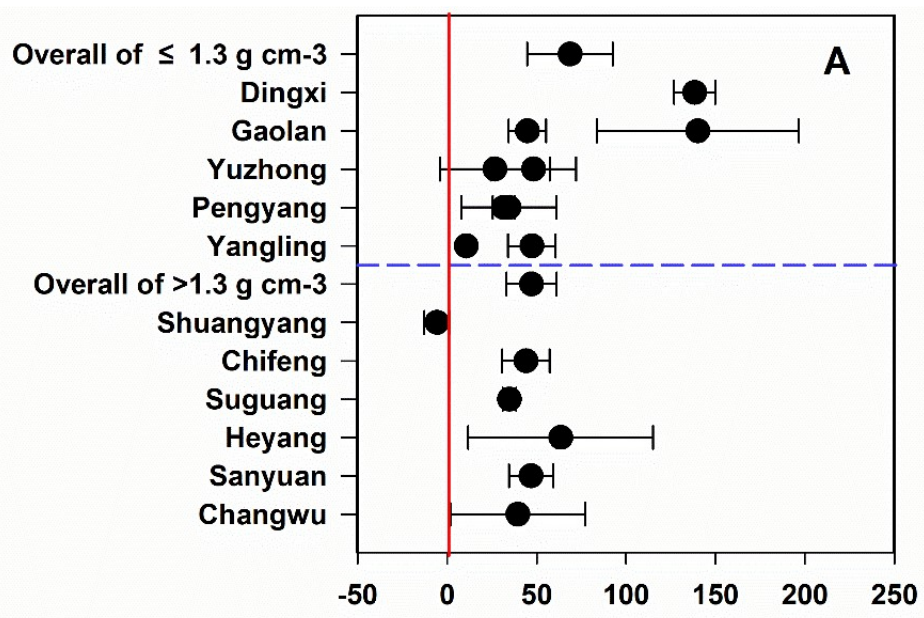

Percentage changes of yield (\%)

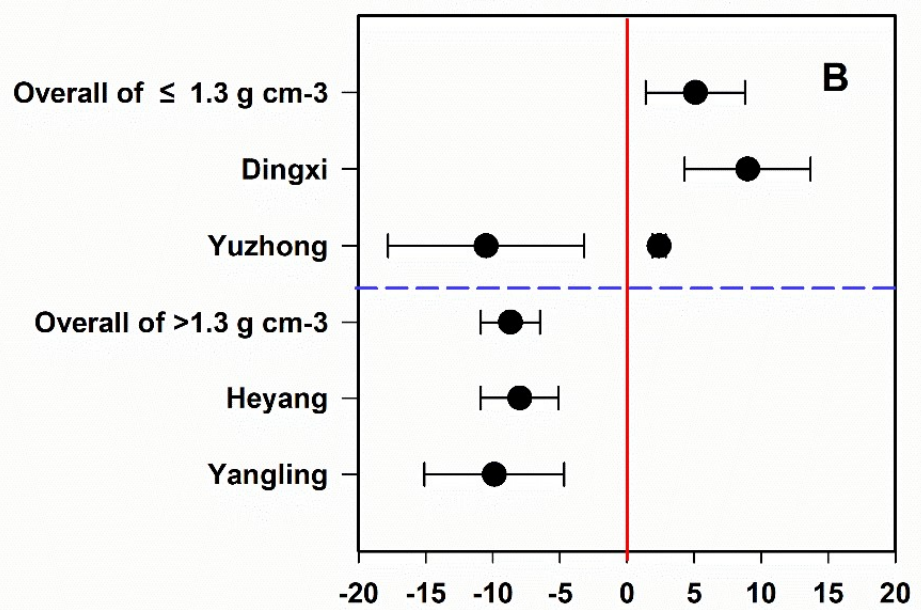

Percentage changes of ET (\%)

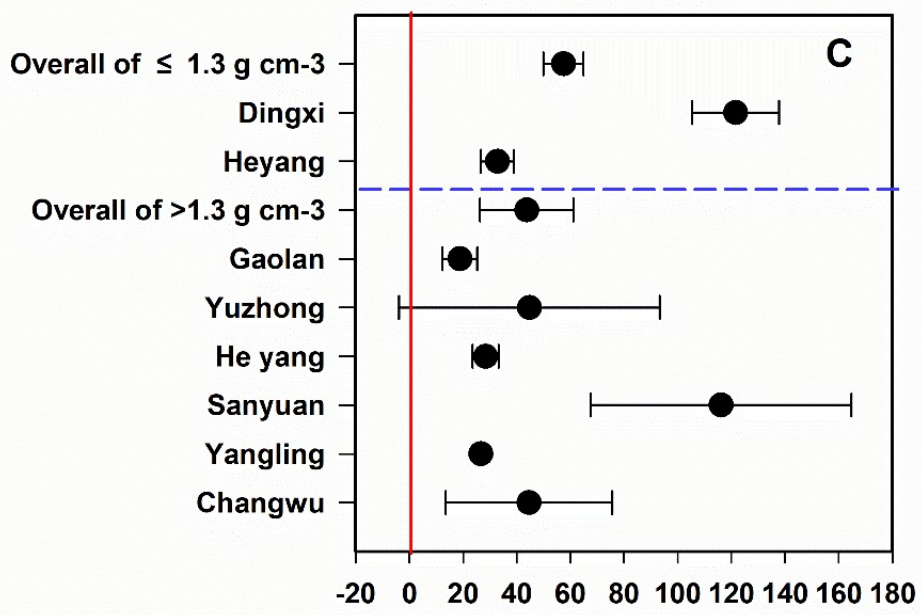

Percentage changes of WUE (\%)

Fig. S6. Percentage changes in maize yield (A), ET (B) and WUE (C) comparing ridgefurrow cultivation to flat-plot cultivation under different soil bulk density. Error bars are the $95 \%$ confidence intervals (CIs). 

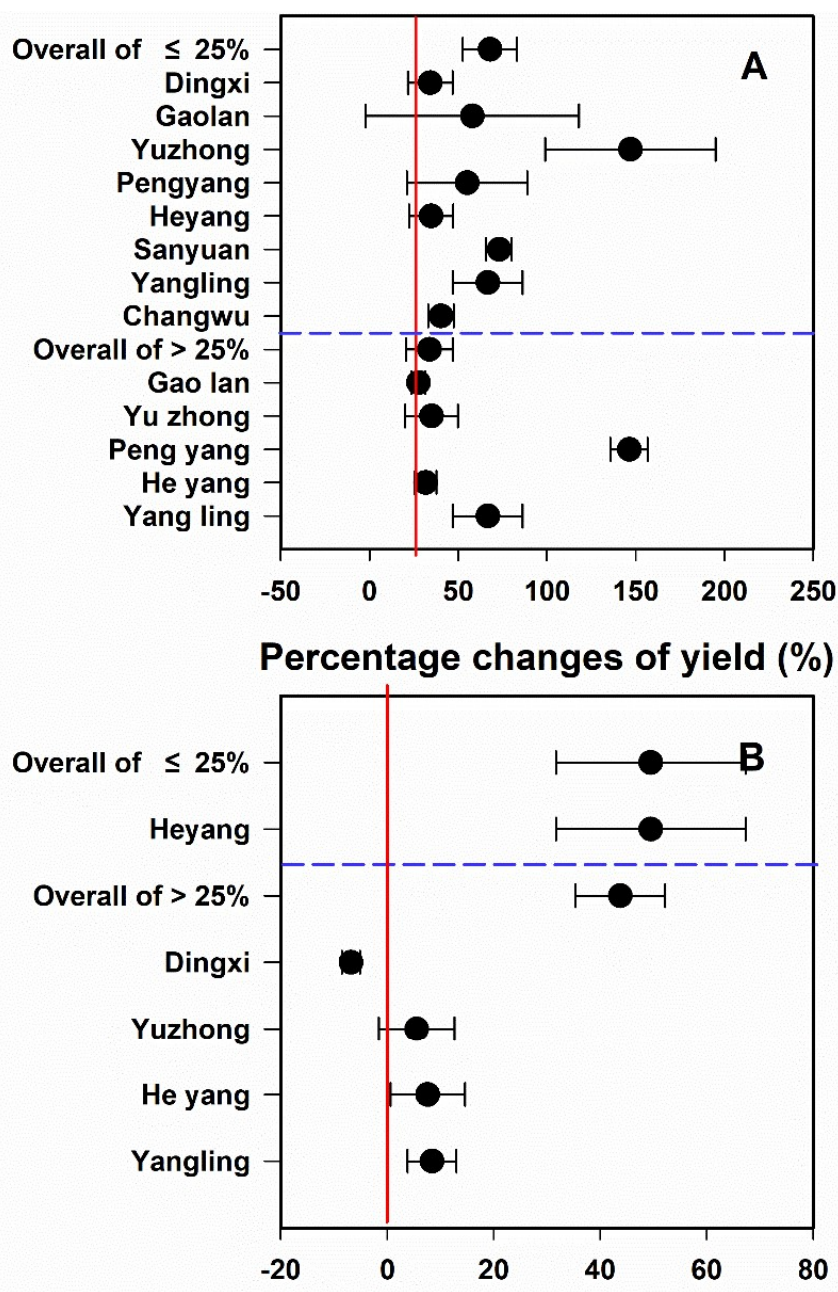

Percentage changes of ET (\%)

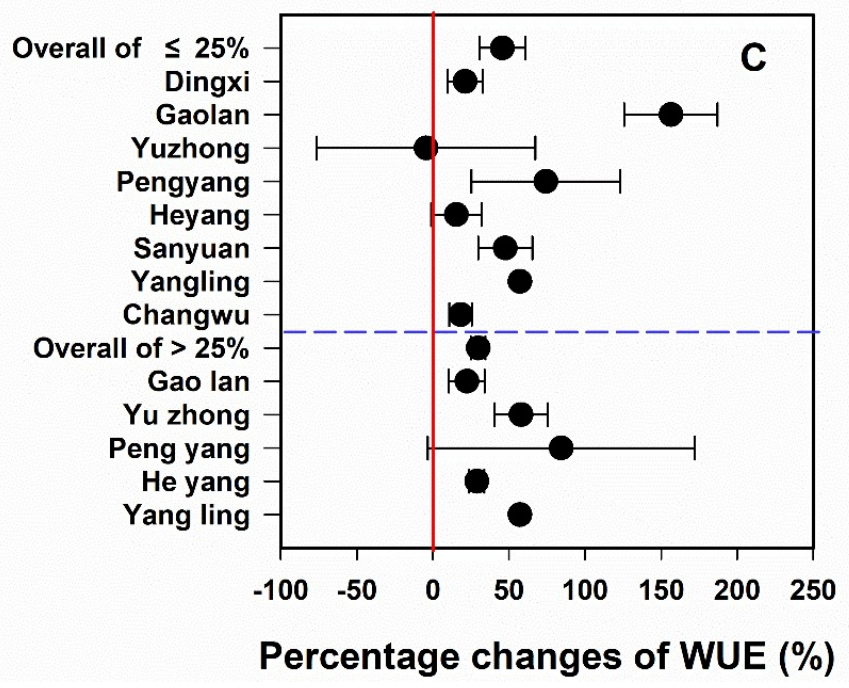

Fig. S7. Percentage changes in maize yield (A), ET (B) and WUE (C) comparing ridgefurrow cultivation to flat-plot cultivation under different field capacity. Error bars are the $95 \%$ confidence intervals (CIs). 


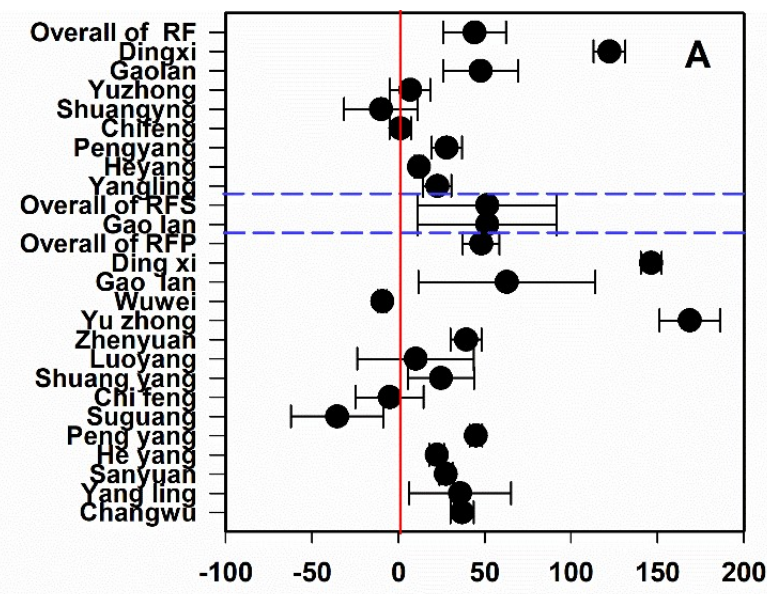

Percentage changes of yield (\%)

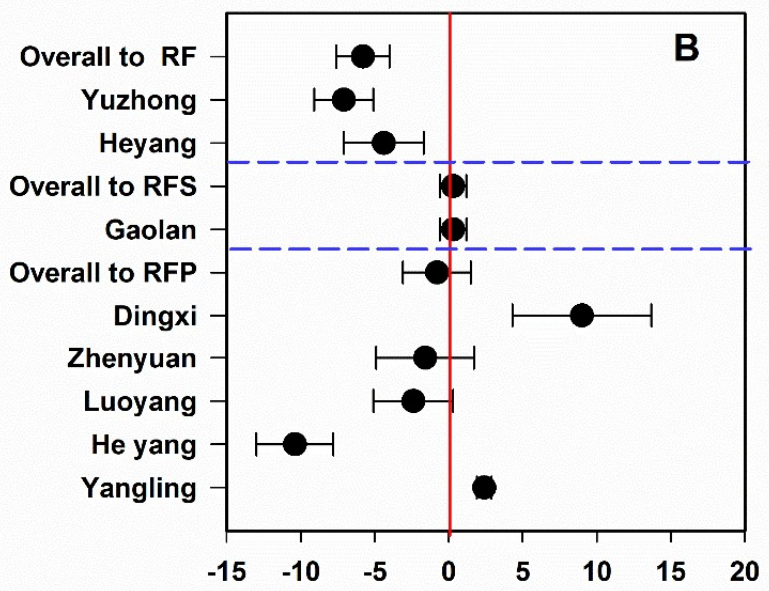

Percentage changes of ET (\%)

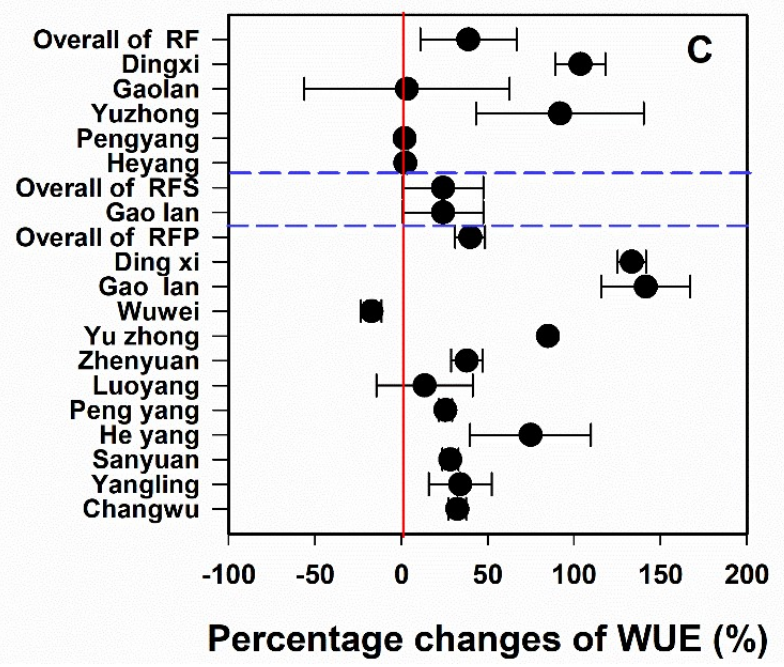

Fig. S8. Percentage changes in maize yield (A), ET (B) and WUE (C) comparing ridgefurrow cultivation to flat-plot cultivation under different mulching management (A, ridge-furrow; B, ridge-furrow mulched with straw; $\mathrm{C}$, ridge-furrow mulched with plastic film). Error bars are the $95 \%$ confidence intervals (CIs). 


\section{S1 Information: References for publications used in the study.}

1. Dong, W., Zhang, L., Duan, Y., Li Sun., Zhao, P., van der Werf, W., Jochem B. Evers, J.B., Wang, Q., Wang, R., Sun, Z., 2017. Ridge and furrow systems with film cover increase maize yields and mitigate climate risks of cold and drought stress in continental climates. Field Crop Res. 207, 71-78.

2. Dong, Q., Yang, Y., Zhang, T., Zhou, L., He, J., Chau, H. W., Zou, Y., Feng, H., 2018a. Impacts of ridge with plastic mulch-furrow irrigation on soil salinity, spring maize yield and water use efficiency in an arid saline area. Agric. Water Manage. 201, 268-277.

3. Dong, W., Yu, H., Wang, R., Wang, Q., Xue, Q., Pan, Z., Sun, Z., Pan, Z., 2018 b. Asymmetric ridge-furrow and film cover improves plant morphological traits and light utilization in rain-fed maize. J. Meteorol. Res. 32(5), 829-838.

4. Eldoma, I.M., Li, M., Zhang, F., Li, F., 2016. Alternate or equal ridge-furrow pattern: which is better for maize production in the rain-fed semi-arid loess plateau of China? Field Crop Res. 191, 131-138.

5. Li, S., Fan, T., Wang, Y., Zhao, G., Wang, L., Tang, X., Dang, Y., Zhao, H., 2014. Effects of plastic film mulching and rain harvesting modes on chlorophyll fluorescence characteristics, yield and water use efficiency of dryland maize. Chin. J. Appl. Ecol. 25, 458-466 (in Chinese).

6. Li, X., Gong, J., Gao, Q., Li, F., 2001. Incorporation of ridge and furrow method of rainfall harvesting with mulching for crop production under semiarid conditions. Agric. Water Manage. 50, 173-183.

7. Li, W., Wen, X., Han, J., Liu, Y., Wu, W., Liao, Y., 2017a. Optimum ridge-to-furrow ratio in ridge-furrow mulching systems for improving water conservation in maize (Zea may L.) production. Environ Sci Pollut Res. 24, 23168-23179.

8. Li, C., Wang, C., Wen, X., Qin, X., Liu, Y., Han, J., Li, Y., Liao, Y., Wu, Y., $2017 b$. Ridge-furrow with plastic film mulching practice improves maize productivity and resource use efficiency under the wheat-maize double-cropping system in dry semi-humid areas. Field Crop Res. 203, 201-211.

9. Liu, T., Chen, J., Wang, Z., Wu, X., Wu, X., Ding, R., Han, Q., Cai, T., Jia, Z., 2018. Ridge and furrow planting pattern optimizes canopy structure of summer maize and obtains higher grain yield. Field Crop Res. 219, 242-249.

10. Qin, X., Li, Y., Han, Y., Hu, Y., Li, Y., Wen, X., Liao, Y., Siddique, K.H.M., 2018. Ridge-furrow mulching with black plastic film improves maize yield more than white plastic film in dry areas with adequate accumulated temperature. Agr Forest Meteorol. 262, 206-214.

11. Ren, X., Jia, Z., Chen, X., Han, Q., Li, R., 2008. Effects of a rainwater-harvesting furrow/ridge system on spring corn productivity under simulated rainfalls. Acta Ecol Sin. 28, 1006-1015.

12. Ren, X., Chen, X., Jia, Z., 2010. Effect of rainfall collecting with ridge and furrow on soil moisture and root growth of corn in semiarid northwest China. J. Agron. Crop Sci. 196, 109-122.

13. Ren, X., Chen, X., Jia, Z., 2016. Impacts of ridge-furrow rainfall concentration systems and mulches on corn growth and yield in the semiarid region of China. $\mathrm{J}$ 
Sci Food Agric. 96, 3882-3889.

14. Ren, X., Chen, X., Cai, T., Wei, T., Wu, Y., Ali, S., Zhang, P., Jia, Z., 2017. Effects of ridge-furrow system combined with different degradable mulching materials on soil water conservation and crop production in semi-humid areas of China. Front. Plant Sci. 8:1877.

15. Tian, Y., Zhao, X., Chen, X., Wang, Z., 2015. The influences of fertilization positions on water and nitrate distribution under wheat/maize ridge and furrow intercropping. Agr Res Arid Area. 33, 72-81(in Chinese).

16. Wang, X., Chen, M., Yi, X., Fu, G., 2009. Effects of ridge width and planting density on corn yields in rainwater-harvesting system with plastic film mulching on ridge. Transactions of the CSAE. 25, 40-47(in Chinese).

17. Wang, H., Zhang, X., Song, S., Ma, Y., Yu, X., Liu, Y., 2011. Effects of whole fieldsurface plastic mulching and planting in furrow on soil temperature, soil moisture, and corn yield in arid area of Gansu Province, Northwest China. Chin. J. Appl. Ecol. 22, 2609-2614(in Chinese).

18. Wang, H., Zhang, X., Song, S., Ma, Y., Yu, X., 2013. Regulation of whole field surface plastic mulching and double ridge-furrow planting on seasonal soil water loss and maize yield in rain-fed area of northwest loess plateau. Sci. Agri. Sin. 46, 917-926(in Chinese).

19. Wu, J., Xiao, K., Zhao, C., Yu, A., Feng, F., Li, L., Chai, Q., 2018. Ridge-furrow cropping of maize reduces soil carbon emissions and enhances carbon use efficiency. Agr. Ecosyst. Environ. 256, 153-162.

20. Zhou, L., Li, F., Jin, S., Song, Y., 2009. How two ridges and the furrow mulched with plastic film affect soil water, soil temperature and yield of maize on the semiarid Loess Plateau of China. Field Crop Res. 113, 41-47.

21. Zhou, L., Jin, S., Liu, C., Xiong, Y., Si, J., Li, X., Gan, Y., Li, F., 2012. Ridgefurrow and plastic-mulching tillage enhances maize-soil interactions: opportunities and challenges in a semiarid agroecosystem. Field Crop Res. 126, 181-188.

22. Zheng, J., Fan, J., Zhang, F., Yan, S., Guo, J., Chen, D., Li, Z., 2018. Mulching mode and planting density affect canopy interception loss of rainfall and water use efficiency of dryland maize on the Loess Plateau of China. J. Arid Land 10(5), 794808 . 